\title{
A HOTELLING MODEL WITH A CEILING ON THE STOCK OF POLLUTION
}

\author{
Ujjayant Chakravorty $^{1, a}$, Bertrand Magné ${ }^{\mathrm{b}}$ and Michel Moreaux ${ }^{\mathrm{c}}$ \\ ${ }^{a}$ Department of Economics, University of Central Florida, Orlando, FL,USA \\ ${ }^{b}$ Université de Toulouse I (CEA, LERNA), 21 Allée de Brienne, 31000 Toulouse, France \\ ${ }^{c}$ Université de Toulouse I (IUF, IDEI and LERNA), 21 Allée de Brienne, 31000 Toulouse, France
}

\begin{abstract}
Environmental agreements such as the Kyoto Protocol aim to stabilize the amount of carbon in the atmosphere, which is mainly caused by the burning of nonrenewable resources such as coal. We characterize the solution to the textbook Hotelling model when there is a ceiling on the stock of emissions. We consider both increasing and decreasing demand for energy. We show that when the ceiling is binding, both the low-cost nonrenewable resource and the high-cost renewable resource may be used jointly. A key implication is that if energy demand were to decline in the long run, we may supplement energy supply through "clean" renewables to meet the environmental standard, but then revert back to using only "dirty" fossil fuels in the future when the ceiling has become non-binding. That is, the much heralded societal "transition" to clean energy resources may be somewhat short-lived.
\end{abstract}

JEL classification : Q12, Q32, Q41

Keywords: Environmental Agreements, Externalities, Fossil Fuels, Nonrenewable Resources, Nonstationary Demand

${ }^{1}$ Corresponding author. Ujjayant Chakravorty, Department of Economics, University of Central Florida, Orlando, FL, 32816-1400. Phone: 407.823.4728. Fax: 407.823.3269. E-mail: uchakravorty@bus.ucf.edu. 


\section{Introduction}

Many nonrenewable resources, such as coal, oil and natural gas, pollute the atmosphere through emissions of carbon, sulphur and other compounds. Environmental agreements such as the Kyoto Protocol have tended to control such pollution by proposing a target pollution concentration, or equivalently, an upper bound on the stock of pollution. ${ }^{2}$ The Montreal Protocol, which aims to phase out the stock of chemicals that deplete the ozone layer, adopts a similar approach, with an eventual goal of a complete phase-out of the stock of these harmful chemicals.

In this paper, we ask how a ceiling on the stock of emissions affects the standard Hotelling model (Hotelling, 1931). We consider a polluting nonrenewable resource and a clean renewable resource. As in Hotelling, it is clear that there will be an eventual transition from the nonrenewable to the renewable resource. What is not so clear is the dynamics of this transition, which is the focus of our analysis.

Little attention has been paid to the problem of how a limit on the stock of emissions may alter the sequence of extraction of a fossil fuel and the backstop resource over time. The theoretical literature has mainly relied on models that specify damage functions caused by the use of nonrenewable resources. ${ }^{3}$ Forster (1980) first examined pollution in a model of nonrenewable resources. Pollution has a negative effect on the utility function, but the clean substitute plays no role except in the terminal phase. Other studies such as Sinclair (1994) and Ulph and Ulph (1994) have examined the time profile of the carbon tax in an infinite horizon framework but without a

\footnotetext{
${ }^{2}$ The Kyoto Protocol (now a treaty) aims at staying below an exogenous upper bound, although country commitments are formulated in terms of emission flows.

${ }^{3} \mathrm{~A}$ ceiling on the stock of emissions may be considered a special case of an increasing and convex damage function for which damages are negligible until the stock reaches a threshold level (the ceiling) and sufficiently high beyond. In general, there is a high degree of uncertainty with respect to the precise shape of the damage function because the actual costs of climate change are difficult to estimate (McKibbin and Wilcoxen, 2002). Another justification for using a ceiling is that most operational climate change models have used ceilings to examine the economic effects of global warming (e.g., see Zhang and Folmer, 1998).
} 
backstop resource. ${ }^{4}$ Hoel and Kverndokk (1996) and Tahvonen (1997) analyze the path of optimal carbon taxes in a model with a nonrenewable resource and a clean backstop. Using stockdependent extraction costs, they show that there may be a period of simultaneous extraction of the nonrenewable and renewable resource. Toman and Withagen (2000) use a general equilibrium framework to examine the role of economic incentives in managing the stock of pollution arising from use of a polluting input but they do not have resource scarcity in their model. Fisher et al. (2004) also do not consider resource scarcity in modeling the relationship between the pollution stock and the development of a clean technology. On the other hand, most empirical work on global warming either assumes a general equilibrium framework that does not explicitly recognize the scarcity of fossil fuels or models the problem by imposing exogenous carbon taxes (e.g., see Manne and Richels, 1991, Nordhaus and Yang, 1996, and Chakravorty et al. 1997).

This paper combines two features that are important in the assessment of the long-run impacts of any international agreement to limit fossil fuel emissions. The first is the scarcity of the nonrenewable resource, which drives up its price over time. The second is the ceiling placed on aggregate emissions from consumption of the resource. As we shall see below, the scarcity of the fossil fuel drives the dynamics of pollution accumulation and the ultimate transition to the cleaner backstop. However, the constraint on the stock of emissions causes the renewable resource to be used even though the cheaper nonrenewable has not yet been exhausted. Once this constraint is no longer binding, the solution reverts to the benchmark Hotelling case.

We first consider stationary demand for energy. We show that the nonrenewable resource is used until the stock of pollution reaches the ceiling. At the ceiling, one of two things may happen. The fossil fuel may be extracted at a constant rate until it becomes scarce and its extraction rate begins to fall. At some point the ceiling ceases to be binding and nonrenewable resource use follows a Hotelling-like path until exhaustion. However, if the cost of the renewable is relatively low, we

${ }^{4}$ Farzin (1996) and Gjerde et al. (1999) address the optimal timing of a carbon tax under threshold effects beyond which the damages become irreversible. 
get an alternative solution in which both resources are extracted simultaneously at the ceiling until the non-renewable resource gets exhausted.

We then examine non-stationary demand. When demand for energy is increasing over time, as is likely to happen in the short run from increases in per capita consumption or growth in population, the sequence of resource use depends upon the relative costs of the nonrenewable and renewable resource, the abundance of the former, whether it is highly (or mildly) polluting, and whether the imposed ceiling is high or low. Only the fossil fuel may be used when the ceiling is binding, or both resources may be used jointly. Or the ceiling may begin with the exclusive use of the nonrenewable and end with joint extraction of both resources. In many of the cases examined, we show that the costly renewable is likely to kick in before the nonrenewable resource is exhausted. These results provide a point of departure from the standard Hotelling notion of a switch from a cheap nonrenewable to a costly renewable resource.

A sharper result is obtained when energy demand decreases over time, which may be a plausible scenario to consider in the long run. ${ }^{5}$ The nonrenewable resource is used at first, then both resources are used at the ceiling, followed again by exclusive use of the nonrenewable resource until it is exhausted and finally, a complete transition to the renewable. That is, the more expensive renewable resource is used at the ceiling even though the cheaper nonrenewable resource has not yet been exhausted. However, when the ceiling is no longer binding, the renewable resource is temporarily abandoned until the nonrenewable resource is completely depleted. ${ }^{6}$

\footnotetext{
${ }^{5}$ For example, demand may decrease if there is a decline in global population and the inevitable levelling off of per capita energy consumption in the developing countries. Recent population projections have significantly downgraded earlier estimates of global population growth and the level from which world population will begin a steady decline (United Nations, 2002, Lutz et al., 2001).

${ }^{6}$ This result violates Herfindahl's (1967) theorem of "least cost first," which suggests that in partial equilibrium, resources must be used in order of increasing extraction cost (see Amigues et al., 1998 for another violation of Herfindahl's theorem).
} 
When emissions can be abated at constant unit cost such as through sequestration by forests or pollution reduction at source, we show that it is never optimal to abate as well as use the renewable resource simultaneously. If the unit cost of abatement is higher than the cost of the renewable resource, there will never be any abatement. In general, however cheap abatement may be, it is never done before the pollution ceiling is attained or when the ceiling ceases to be binding. Abatement takes place only at the ceiling. The timing of abatement depends on how demand may be changing with time. When demand is stationary or decreasing, abatement begins exactly when the stock of emissions reaches the ceiling. When demand is increasing, the period of abatement may be at the beginning or the end of the ceiling period, or in the strict interior.

The analysis suggests that if an agreement such as the Kyoto Protocol were to be implemented, we may see the joint use of fossil fuels and renewable energy. Renewables such as solar energy may be employed in electricity generation even though they are costly relative to coal. However, if global populations and energy demand peak and then begin a steady decline, the pollution ceiling may not be binding any longer. We may then abandon the expensive solar energy and revert back to using fossil fuels exclusively. The relative abundance of coal over the other fossil fuels (oil and natural gas) may suggest that the second period with exclusive use of coal may be an extended one. Renewable energy may be used in the short run to complement fossil fuels and meet environmental targets, but they may be abandoned in the long run when energy demand falls.

Section 2 describes the Hotelling model with an exogenous ceiling on the stock of pollution. Section 3 develops intuition by focusing on stationary demand. Sections 4 and 5 deal with increasing and decreasing demand, respectively. Section 6 concludes the paper.

\section{The Hotelling Model with a Ceiling on the Stock of Pollution}

Suppose the economy uses two resources for power generation: a polluting non-renewable resource (e.g., a fossil fuel such as coal) and a clean renewable resource (e.g., solar energy). For ease of exposition we will call them coal and solar energy, respectively. They are perfect substitutes. If $x_{t}$ and $y_{t}$ are their respective extraction (consumption) rates, then aggregate energy consumption at time $t$ is given by $q_{t}=x_{t}+y_{t}$. The instantaneous gross surplus or utility from 
energy consumption at time $t$ is $u\left(q_{t}, t\right)$. We assume that $u: R_{+}^{2} \rightarrow R_{++}$is of class $\mathbf{C}^{3}$, strictly increasing and strictly concave in $q$, i.e., $u_{1}\left(q_{t}, t\right) \equiv \delta u\left(q_{t}, t\right) / \delta q_{t}>0$ and $u_{11}\left(q_{t}, t\right) \equiv \delta^{2} u\left(q_{t}, t\right) / \delta q_{t}^{2}<0, q_{t}>0, t>0$.

The short-run scenario in which energy demand is increasing over time is denoted $I D$ and is compared to stationary demand $(S D)$ and decreasing demand $(D D) .{ }^{7}$ Demand is increasing (stationary, decreasing) if utility and marginal utility are both increasing (constant, decreasing) and if $u$ is bounded from above (below) by $\bar{u}(\underline{u})$. That is, for any $q_{t}>0, t \geq 0$, $u_{2}\left(q_{t}, t\right) \equiv \partial u\left(q_{t}, t\right) / \partial t>0(=,<0), u_{12}\left(q_{t}, t\right) \equiv \partial^{2} u\left(q_{t}, t\right) / \partial q_{t} \partial t>0(=,<0)$ and $\lim _{t \uparrow+\infty} u\left(q_{t}, t\right)=\bar{u}\left(q_{t}\right)\left(\underline{u}\left(q_{t}\right)\right)$.

We also make the following regularity assumption which will be useful later. For $I D(S D, D D)$, given any $q_{t}>0, t \geq 0$, let $\partial^{3} u\left(q_{t}, t\right) / \partial q_{t} \partial t^{2} \equiv u_{122}\left(q_{t}, t\right)<0(=,>0)$. This implies that the marginal utility at a given $q_{t}$ is an increasing and concave function of time for $I D$ and a decreasing and convex function for $D D$. These assumptions suggest that when demand is increasing, marginal utility must increase over time $\left(u_{12}>0\right)$, but at a decreasing rate $\left(u_{122}<0\right)$. The restriction on the upper bound of utility suggests that aggregate energy consumption is bounded. It will be satisfied if aggregate population and per capita energy consumption are bounded from above. In the decreasing demand case, the above assumptions imply marginal utility of energy consumption decreasing at an increasing rate.

Initial reserves of coal are assumed known and denoted $X_{0}$, the constant average extraction cost ${ }^{8}$ of coal to the user is $c_{e}$ and the pollution per unit of coal consumed is given by $\zeta$. Let $X_{t}$ be the amount of coal available at time $t$, so that $\dot{X}_{t}=-x_{t}$. Coal is assumed to be scarce even in the $D D$ case. That is, let $x_{c t}$ be the quantity such that the marginal utility from coal is equal to its marginal cost, $u_{1}\left(x_{c t}, t\right)=c_{e}$. Then scarcity implies that the stock necessary to sustain this path (infinite for

\footnotetext{
${ }^{7}$ The latter may happen with a decline in world population in the long run so that aggregate energy consumption falls even with the expected increases in per capita energy demand in the developing countries.

${ }^{8}$ The extraction cost is essentially a delivery cost, i.e., includes processing and transportation.
} 
both $S D$ and $I D$ ) is higher than $X_{0}$. Mathematically, $\lim _{t \uparrow+\infty} \int_{0}^{t} x_{c \tau} d \tau>X_{0}$.

Let $Z_{t}$ be the stock of pollution. The gross emission of pollution $z_{t}$ is assumed to be proportional to the consumption of coal, $z_{t}=\zeta x_{t}$, where $\zeta$ is some constant. As is standard in the literature, we also assume that the natural regeneration capacity of the atmosphere is proportional to the stock of pollution $Z_{t}$ (see e.g., Kolstad and Krautkraemer, 1993). However, the stock of pollution can be reduced through costly abatement. Let $a_{t}$ be the instantaneous rate of abatement. Then the rate of change of $Z_{t}$ is given by $\dot{Z}_{t}=\zeta x_{t}-a_{t}-\alpha Z_{t}, \alpha>0, t \geq 0$. Let $c_{a}$ be the unit cost of abatement assumed to be constant so that the total abatement cost at time $t$ is $c_{a} a_{t \cdot}{ }^{9}$

Define $\bar{Z}$ to be the ceiling on the pollutant stock imposed exogenously by the regulator. This may be the outcome of an environmental agreement such as the Kyoto Protocol. Then $\bar{Z}-Z_{t} \geq 0$. Let $\bar{x}\left(a_{t}\right)$ be the maximum consumption rate of coal if $Z_{t}$ is at its upper bound $\bar{Z}$, and abatement is equal to $a_{t}$. That is, $\bar{x}\left(a_{t}\right)=\left(a_{t}+\alpha \bar{Z}\right) / \zeta$. Let $\bar{p}_{e t}\left(a_{t}\right)$ be the corresponding marginal utility defined by $\bar{p}_{e t}\left(a_{t}\right)=u_{1}\left(\bar{x}\left(a_{t}\right), t\right)$. Since $\partial \bar{p}_{e t}\left(a_{t}\right) / \partial t=u_{12}\left(\bar{x}\left(a_{t}\right), t\right)$ and $\partial^{2} \bar{p}_{e t}\left(a_{t}\right) / \partial t^{2}=u_{122}\left(\bar{x}\left(a_{t}\right), t\right)$, then for any given $a_{t}, \bar{x}\left(a_{t}\right)$ is increasing and concave for $I D$, constant for $S D$, and decreasing and convex for $D D$. When there is no active abatement, $\bar{x}(0)$ and $\bar{p}_{e t}(0)$ are simply denoted by $\bar{x}$ and $\bar{p}_{e t}$, respectively. If $\bar{p}_{e t}<c_{r}$ for $I D$, or if $\bar{p}_{e t}>c_{r}$ for $D D$, we denote by $\eta$ the date at which $\bar{p}_{e t}=c_{r}$, if such a time exists.

Let $\bar{y}$ be the instantaneous rate of solar energy available. It is nonstorable so that the part $\bar{y}-y_{t}$ which is not immediately used, is lost. Define $c_{r}$ as the constant average delivery cost of solar energy. This cost is higher than the cost of coal, $c_{r}>c_{e}$. We assume that the marginal utility at small consumption rates is higher than $c_{r}$ even for $D D$, so that solar energy has to be used once

\footnotetext{
${ }^{9}$ Abatement can be done in two ways. Carbon emissions from coal can be reduced at source through scrubbing. Alternatively, the stock can be reduced through increasing the forest area. We abstract from these considerations by assuming that the cost per unit abatement is the same for both methods, and equals $c_{a}$.
} 
coal is exhausted, and as we shall show, sometimes before. That is, there exists an $\varepsilon>0$ such that $\lim _{t \uparrow+\infty} u_{1}\left(q_{t}, t\right) \geq c_{r}+\varepsilon, t \geq 0$.

For convenience, solar energy is assumed to be abundant even for ID. Define $y_{c t}$ as the consumption rate for which the marginal utility equals the marginal cost of solar energy, $u_{1}\left(y_{c t}, t\right)=c_{r}, t \geq 0$. Then $y_{c t}$ is time increasing for $I D$, constant for $S D$, and decreasing for $D D$. The abundance assumption could be written as $y_{c t} \leq \bar{y}, t \geq 0$. ${ }^{10}$

\section{The Optimization Problem}

The social planner maximizes welfare by choosing the quantities of coal and solar energy as well as carbon abated at any given instant:

$$
\begin{aligned}
\max _{\left\{\left(x_{t}, y_{t}, a_{t}\right), t \geq 0\right\}} \int_{0}^{+\infty}\left\{u\left(x_{t}+y_{t}, t\right)-c_{e} x_{t}-c_{a} a_{t}-c_{r} y_{t}\right\} e^{-\rho t} d t \\
\text { s.t. } \quad \dot{X}_{t}=-x_{t}, X_{0} \text { given, } X_{t} \geq 0, \\
x_{t} \geq 0, \\
a_{t} \geq 0, \\
\dot{Z}_{t}=\zeta x_{t}-a_{t}-\alpha Z_{t}, Z_{0}<\bar{Z} \text { given, } \bar{Z}-Z_{t} \geq 0, \\
y_{t} \geq 0 .
\end{aligned}
$$

The Lagrangian $L_{t}$ is given by

\footnotetext{
${ }^{10}$ Since the focus of the paper is on the effect of the ceiling on the extraction path of the nonrenewable and renewable resource, we consider the simple case of an abundant renewable resource available at constant cost. This excludes plausible backstop technologies such as nuclear power, which itself is generated from a nonrenewable resource (uranium) that can also be recycled. Including these features will make our model complicated and so this task is left for future work. For an empirical model with nuclear power see Chakravorty, Magné and Moreaux (2005).
} 


$$
\begin{aligned}
L_{t}= & u\left(x_{t}+y_{t}, t\right)-c_{e} x_{t}-c_{a} a_{t}-c_{r} y_{t}-\lambda_{t} x_{t}+\mu_{t}\left[\zeta x_{t}-a_{t}-\alpha Z_{t}\right] \\
& +v_{t}\left[\bar{Z}-Z_{t}\right]+\gamma_{e t} x_{t}+\gamma_{a t} a_{t}+\gamma_{r t} y_{t},
\end{aligned}
$$

and the first order conditions are

$$
\begin{aligned}
& \frac{\partial L_{t}}{\partial x_{t}}=0 \quad \Leftrightarrow \quad u_{1}-c_{e}-\lambda_{t}+\zeta \mu_{t}+\gamma_{e t}=0, \\
& \frac{\partial L_{t}}{\partial y_{t}}=0 \quad \Leftrightarrow \quad u_{1}-c_{r}+\gamma_{r t}=0, \quad \text { and } \\
& \frac{\partial L_{t}}{\partial a_{t}}=0 \quad \Leftrightarrow \quad-c_{a}-\mu_{t}+\gamma_{a t}=0,
\end{aligned}
$$

together with the complementary slackness conditions

$$
\begin{aligned}
& v_{t} \geq 0, \text { and } v_{t}\left[\bar{Z}-Z_{t}\right]=0, \\
& \gamma_{e t} \geq 0, \text { and } \gamma_{e t} x_{t}=0, \\
& \gamma_{r t} \geq 0, \text { and } \gamma_{r t} y_{t}=0, \text { and } \\
& \gamma_{a t} \geq 0, \text { and } \gamma_{a t} a_{t}=0 .
\end{aligned}
$$

The dynamics of the costate variables are determined by

$$
\begin{aligned}
& \dot{\lambda}_{t}=\rho \lambda_{t}-\frac{\partial L_{t}}{\partial X_{t}} \Leftrightarrow \dot{\lambda}_{t}=\rho \lambda_{t} \Rightarrow \lambda_{t}=\lambda_{0} e^{\rho t}, \text { and } \\
& \dot{\mu}_{t}=\rho \mu_{t}-\frac{\partial L}{\partial Z_{t}} \Leftrightarrow \dot{\mu}_{t}=(\rho+\alpha) \mu_{t}+v_{t} .
\end{aligned}
$$

The costate variable $\mu_{t}$ is non-positive. If $Z_{t}<\bar{Z}$ over some time interval $\left[t_{1}, t_{2}\right]$, then $v_{t}=0$ over that interval and $\mu_{t}=\mu_{t_{1}} e^{(\rho+\alpha)\left(t_{1}-t\right)}, t \in\left[t_{1}, t_{2}\right]$. Lastly, the transversality conditions at infinity are given by 


$$
\lim _{t^{\uparrow}+\infty} e^{-\rho t} \lambda_{t} X_{t}=\lambda_{0} \lim _{t^{\uparrow}+\infty} X_{t}=0
$$

and

$$
\lim _{t \uparrow+\infty} e^{-\rho t} v_{t} Z_{t}=0
$$

\section{Price Paths for Coal}

It is useful to obtain some insight by considering the "pure" Hotelling price path. For any initial scarcity rent $\lambda_{0} \in\left(0, c_{r}-c_{e}\right)$, let $\tilde{x}_{t}$ be the solution to the equation $u_{1}\left(x_{t}, t\right)=c_{e}+\lambda_{0} e^{\rho t} \equiv \tilde{p}_{t}$, if it exists, zero otherwise. ${ }^{11}$ Then $\tilde{x}_{t}$ is the extraction rate of coal with the ceiling non-binding at time $t$ and never tight at any time $\tau>t$, provided that $\tilde{p}_{t}<c_{r}$ and $\lambda_{0}$ is the right initial scarcity rent. The time derivative of $\tilde{x}_{t}$ cannot be signed for $I D$ since $\frac{\partial \tilde{x}_{t}}{\partial t}=\frac{-u_{12}\left(\tilde{x}_{t}, t\right)+\rho \lambda_{0} e^{\rho t}}{u_{11}\left(\tilde{x}_{t}, t\right)}$ which is indeterminate. But $\partial \tilde{x}_{t} / \partial t<0$ for $S D$ and $D D$. Extraction must decline with an increase in price under stationary and decreasing demand. Further, $\tilde{x}_{t}$ decreases with $\lambda_{0}, \partial \tilde{x}_{t} / \partial \lambda_{0}<0$ and $\lim _{\lambda_{0} \downarrow 0} \tilde{x}_{t}=x_{c t}$

Define $\tilde{\theta}$ as the time at which the Hotelling price equals the cost of solar energy, i.e., $\tilde{p}_{t}=c_{r}$. Then $\tilde{\theta}=\rho^{-1}\left[\log \left(c_{r}-c_{e}\right)-\log \lambda_{0}\right]$. We denote by $\tilde{\tau}$ the time at which $\tilde{p}_{t}=\bar{p}_{e t}{ }^{12}$ Absent the ceiling constraint, the optimal value of $\lambda_{0}$, denoted by $\lambda_{0}^{f}$, is the solution to the cumulative demand/supply balance equation $\int_{0}^{\tilde{\theta}} \tilde{x}_{t}\left(\lambda_{0}\right) d t=X_{0}$.

Let $\tilde{Z}_{t}$ be the pollution stock induced by $\tilde{x}_{t}$ when the initial stock is at $Z_{0}$. That is

\footnotetext{
${ }^{11}$ This caveat holds for future definitions and is not repeated.

${ }^{12}$ In general, we use $\theta$ to denote the time at which a price or a marginal cost equals $c_{r}$ and $\tau$ the time at which a price or a marginal cost equals $\bar{p}_{e t}$. When $\bar{p}_{e t}=c_{r}$, the corresponding time is denoted by $\delta$.
} 
$d \tilde{Z}_{t} / d t=\zeta \tilde{x}_{t}-\alpha \tilde{Z}_{t}$ and $\tilde{Z}_{0}=Z_{0}$. Then $\tilde{Z}_{t}\left(\lambda_{0}^{f}\right)$ is the trajectory of the pollution stock in the time interval $\left[0, \tilde{\theta}\left(\lambda_{0}^{f}\right)\right]$ in the absence of the ceiling constraint. Beyond time $\tilde{\theta}\left(\lambda_{0}^{f}\right)$, no coal is used so emissions are zero and the stock $Z_{t}$ declines to zero. In what follows, we assume that the ceiling constraint is always binding along the standard Hotelling path, that is $\max \left\{\tilde{Z}_{t}\left(\lambda_{0}^{f}\right), t \in\left[0, \tilde{\theta}\left(\lambda_{0}^{f}\right)\right]\right\}>\bar{Z}$. The extraction path $\tilde{x}_{t}$ is not optimal at the beginning of the planning horizon.

When abatement occurs, $\mu_{t}=-c_{a}+\gamma_{a t}$ by (4). Suppose the ceiling constraint is binding, then we have $Z_{t}=\bar{Z}$. If $x_{t}>\bar{x}$, then there must be abatement of surplus emissions so that $a_{t}$ equals $\zeta\left(x_{t}-\bar{x}\right)$ and $\gamma_{a t}=0$ from (8). Thus $\mu_{t}=-c_{a}$. From (6), $\gamma_{e t}=0$ so using (9) and substituting into (2), the social marginal cost of a unit of coal $u_{1}\left(x_{t}, t\right)$ is given by $c_{e}+\zeta c_{a}+\lambda_{0} e^{\rho t}$. Call this $\breve{p}_{t}$, with $\breve{x}_{t}$ as the solution to $u_{1}\left(x_{t}, t\right)=\breve{p}_{t}$. Then $\breve{x}_{t}$ is the optimal extraction rate of coal if at time $t$ the ceiling is binding, part of the emission flow is abated and $\breve{p}_{t}<c_{r}$. The derivative of $\breve{x}_{t}$ with respect to time cannot be signed for $I D$, is zero for $S D$ and negative for $D D$.

Define $\breve{\theta}$ as the time at which the above price $\breve{p}_{t}=c_{r}$ so that $\breve{\theta}=\rho^{-1}\left[\log \left(c_{r}-c_{e}-\zeta C_{a}\right)-\log \lambda_{0}\right]$. Let $\breve{\tau}$ be the time at which $\breve{p}_{t}=\bar{p}_{e t}$, if such a time exists and is unique over [0, $\left.\infty\right)$. If two such instants of time exist, we denote them by $\breve{\tau}_{1}$ and $\breve{\tau}_{2}$, with $\breve{\tau}_{1}<\breve{\tau}_{2}$. When the ceiling is not binding but will in the future, $Z_{t}<\bar{Z}$ and by (5), $v_{t}=0$ so that from (10) the absolute value of $\mu_{t}$ must grow at the rate $\rho+\alpha$. From (6), $\gamma_{e t}=0$. Then for $\lambda_{0} \in\left[0, c_{r}-c_{e}\right]$ and $\mu_{0} \in\left(-\left[c_{r}-\left(c_{e}+\lambda_{0}\right)\right] / \zeta, 0\right)$, define $\hat{p}_{t}$ as the corresponding marginal cost of coal where from (2), we have $\hat{p}_{t}=c_{e}+\lambda_{0} e^{\rho t}-\zeta \mu e^{(\rho+\alpha) t}$. Let $\hat{x}_{t}$ be the extraction rate for coal at which the marginal utility equals this marginal cost, i.e., that is, $\hat{x}_{t}$ is the solution to $u_{1}(x, t)=\hat{p}_{t}$. Thus $\hat{x}_{t}$ is the optimal extraction rate of coal if at time $t$ the ceiling is not binding, has never been earlier, but will be in the future. The time derivative of $\hat{x}_{t}$ cannot be signed for $I D$ but is negative for $S D$ and $D D$. 
Denote by $\hat{\theta}$ the time at which $\hat{p}_{t}=c_{r}$. Then $\hat{\theta}=\rho^{-1}\left[\log \left(c_{r}-c_{e}\right)-\log \left(\lambda_{0}-\zeta \mu_{0}\right)\right]$. Define $\hat{Z}_{t}$ as the pollution stock generated by $\hat{x}_{t}$, starting from $Z_{0}$, so that $\partial \hat{Z}_{t} / \partial t=\zeta \hat{x}_{t}-\alpha \hat{Z}_{t}$ and $\hat{Z}_{0}=Z_{0}$. For any $\lambda_{0} \in\left[0, \bar{p}_{e 0}-c_{e}\right]$ and $\mu_{0} \in\left(-\left[\bar{p}_{e 0}-\left(c_{e}+\lambda_{0}\right)\right] / \zeta, 0\right)$, let $\hat{\tau}$ be the time at which $\hat{p}_{t}=\bar{p}_{e t}$. Lastly, for any $\lambda_{0} \in\left[0, \bar{p}_{e 0}-c_{e}\right]$ and $\mu_{0} \in\left(-\left[c_{r}-\left(c_{e}+\lambda_{0}\right)\right] / \zeta, 0\right)$, denote by $\delta$ the time at which $\hat{p}_{t}=\breve{p}_{t}$. Under the regularity assumptions, $\delta$ is well-defined and unique.

\section{The Stationary Demand Case}

In order to develop intuition, we first explore the simple case of stationary demand. When demand does not change with time, $\bar{p}_{e t}, \bar{x}_{t}$ and $y_{c t}$ are constant, so the time subscript can be deleted. There are two cases to consider, $\bar{p}_{e}<c_{r}$ and $\bar{p}_{e}>c_{r}$.

Case $\bar{p}_{e}<c_{r}$

The price of coal at the ceiling when there is no abatement is equal to $\bar{p}_{e}$. The inequality condition suggests that the price of energy will never exceed $c_{r}$, since the entire demand at price $c_{r}$ can be met by the clean backstop, as shown in fig.1. However, the price of energy may exceed $\bar{p}_{e}$ in which case only coal may be used. The condition $\bar{p}_{e}<c_{r}$ may occur if coal is relatively clean, energy demand is low or the ceiling is high. If the abatement cost $c_{a}$ is low enough, then abatement must be done over some time interval during which the ceiling constraint is tight. There is no gain from abating when the stock is strictly below the ceiling. Specifically, abatement occurs iff $\tilde{p}_{t}=\bar{p}_{e}$ sufficiently early in the time horizon. If not, the incremental cost of abatement $\zeta c_{a}$ would be higher than the marginal utility at the ceiling over the entire time period. It will not be optimal to abate.

[Fig. 1 here]

The optimal sequence of resource use has five phases. Since $Z_{0}<\bar{Z}$, the ceiling is non-binding in the first phase and only coal is used. The ceiling constraint binds in the future so in the first phase the shadow price $-\mu_{t}$ grows at the rate $\rho+\alpha$, and $p_{t}=\hat{p}_{t}$. The quantity of coal extracted is 
more than at the ceiling, $\hat{x}_{t}>\bar{x}$, so emissions exceed natural dilution hence the pollution stock accumulates over time. At the end of this period, the ceiling is attained. We then have $\hat{p}_{t}=\breve{p}_{t}$, since the price path must be continuous.

In the second phase given by the time interval $[\delta, \widetilde{\tau})$, it is economical to abate since $\breve{p}_{t}<\bar{p}_{e}$. The price of coal is given by $\breve{p}_{t}$. Extraction is higher than $\bar{x}, \breve{x}_{t}>\bar{x}$ and the excess is abated, $a_{t}=\left(\breve{x}_{t}-\bar{x}\right)$. At time $\bar{\tau}, \breve{p}_{t}=\bar{p}_{e}$ and abatement becomes too costly. In the third phase $[\bar{\tau}, \tilde{\tau})$, there is no abatement and only coal is used at the ceiling, $q_{t}=\bar{x}$. The price of coal is constant at $\bar{p}_{e}$.

In the next phase the ceiling is no longer relevant, so resource use is strictly Hotelling starting from the residual stock at time $\tilde{\tau}$. In the interval $[\tilde{\tau}, \tilde{\theta}), p_{t}=\tilde{p}_{t}$, coal price increases and extraction declines from the ceiling level. At time $\tilde{\tau}$, the stock is at the ceiling but with declining extraction, the ceiling constraint is no more binding. In this phase, $\mu_{t}=0$ and the price of coal is pure Hotelling, given by $c_{e}+\lambda_{0} e^{\rho t}$. At time $\tilde{\theta}$ coal is exhausted while the use of solar energy jumps from 0 to $y_{c}$ to ensure continuity of the price path. In the terminal phase $[\tilde{\theta},+\infty)$, only solar energy is used.

Even if the unit cost of abatement $c_{a}$ is small (but not zero), we must have a phase during which coal is used at the ceiling but there is no abatement. This is an immediate implication of the fact that the $\breve{p}_{t}$ curve is a vertical translation of the $\tilde{p}_{t}$ curve by the distance $\zeta c_{a}$. Thus $\widetilde{\tau}<\tilde{\tau}$ and the phase $[\widetilde{\tau}, \tilde{\tau}]$ never disappears, except in the limiting case when $c_{a}=0$. Of course, if abatement costs are zero, the ceiling itself is of no relevance and the entire extraction program will proceed according to Hotelling. Excess emissions will be abated costlessly.

If abatement cost $c_{a}$ is high enough, the curve $\breve{p}_{t}$ will lie above the $\bar{p}_{e}$ line over the time interval $[0, \hat{\tau})$ during which $\hat{p}_{t}<\bar{p}_{e}$ (see fig. 1 ). Then the marginal cost of coal with abatement is always 
higher than the marginal utility of consumption even when the pollution stock is constrained. In this case the phase $[\delta, \widetilde{\tau})$ during which the ceiling is binding and abatement occurs will disappear. The solution for this case is formally presented in Appendix A. ${ }^{13}$

\section{Case $\bar{p}_{e}>c_{r}$}

In this case solar energy becomes economical at a price below the price of energy when the ceiling is tight and there is no abatement (see fig. 2). The demand for energy at $c_{r}$ is higher then at the ceiling price $\bar{p}_{e}$. This implies that the price $\bar{p}_{e}$ will never be reached since solar energy is already economical at a lower price. In fact the price of energy will never exceed the price of the backstop $c_{r}$. As in the previous case, if abatement cost $c_{a}$ is sufficiently low, abatement begins at time $\delta$, the start of the ceiling period. With abatement, coal can be used at a rate higher than $\bar{x}$. Abatement ends at time $\breve{\theta}$ when $\breve{p}_{t}=c_{r}$ and solar energy becomes competitive. Abatement is replaced by use of the renewable resource, while coal continues to be extracted at the maximum rate $\bar{x}$. This phase ends at time $\tilde{\theta}$ when coal is exhausted and the consumption of solar energy jumps from $y_{c}-\bar{x}$ to $y_{c}$ while maintaining the continuity of the price path. Observe that in this model, once abatement is complete, the ceiling price must be at $c_{r}$, since at lower prices, coal alone cannot satisfy the entire demand. Another general observation is that abatement of emissions and use of solar energy are strict substitutes and cannot be used jointly over any nondegenerate interval of time. This is because the cost of energy with abatement rises over time while the cost of solar energy is constant. They cannot be equal except at a point.

[Fig. 2 here]

\section{Increasing Demand for Energy in the Short Run}

Global demand for energy is increasing because of population growth and increases in consumption per capita, mainly in the developing countries. In this section we examine this short run scenario of increasing demand. Now $\bar{p}_{e t}$ is no longer constant but is increasing over time and

\footnotetext{
${ }^{13}$ The technical statement of the solutions discussed here are given in Appendix A. Here we focus on the graphical exposition.
} 
concave. We classify according to whether $\bar{p}_{e t}$ is everywhere lower than $c_{r}$, cuts it from below, or everywhere higher than $c_{r}$. We consider these cases below.

\section{Case $\bar{p}_{e \infty}<c_{r}$}

The two possible solutions are illustrated in figs. 3 and 4 for sufficiently low unit abatement costs. The solution in fig. 3 is similar to the one shown in fig. 1 except that because of increasing demand, during the interval $[\widetilde{\tau}, \tilde{\tau}$ ) when the ceiling is binding and there is no abatement, the price $\bar{p}_{e t}$ is increasing. However the quantity of coal extracted during this phase must be constant since the ceiling binds. At the ceiling given by the time interval $[\delta, \tilde{\tau})$, there are two phases, one with active abatement followed by no abatement.

[Fig. 3 here]

The case shown in fig. 4 only occurs under increasing demand, as will become clear later. The time interval $[\delta, \tilde{\tau})$ during which the ceiling binds is split into three: a first and a third phase with no abatement, and a middle phase $\left[\breve{\tau}_{1}, \breve{\tau}_{2}\right)$ with active abatement. This solution can not occur in the SD case because there the $\bar{p}_{e t}$ curve is constant and the price with abatement $\breve{p}_{t}$ must occur at the beginning of the ceiling period, if at all (see fig. 1).

[Fig. 4 here]

If unit abatement cost $c_{a}$ is low, the distance between the curves $\breve{p}_{t}$ and $\tilde{p}_{t}$, given by $\zeta c_{a}$, is small. In the first phase where the price of coal is given by $\hat{p}_{t}$ and the pollution stock is accumulating, the $\hat{p}_{t}$ curve will intersect the $\breve{p}_{t}$ curve before intersecting the $\bar{p}_{e t}$ curve (see fig. 4). For a given $\bar{p}_{e t}$ curve and $\zeta c_{a}$ sufficiently low, $\breve{p}_{0}=\bar{p}_{e \infty}$ and at the same time $\hat{p}_{0}<\breve{p}_{0}$. Thus for sufficiently low abatement costs, the best policy is to abate immediately when the ceiling constraint is binding. As in the $S D$ case, even if $c_{a}$ is very small, as long as it is positive, there must exist a second phase at the ceiling without abatement during which the price of coal equals $\bar{p}_{e t}$. 
These two phases at the ceiling may occur up to some critical value of unit abatement cost beyond which this initial abatement phase disappears (see fig. 3). We may then have the three phase pattern at the ceiling depicted in fig. 4 , where abatement occurs strictly interior to the ceiling period. For even higher values of $c_{a}$, this intermediate phase with abatement, $\left[\breve{\tau}_{1}, \breve{\tau}_{2}\right)$ itself may disappear and abatement may occur at the end of the ceiling period as in fig. 7 . There exist two critical values of the abatement cost denoted by $\bar{c}_{a}^{\prime}$ and $\bar{c}_{a}^{\prime \prime}$, with $\bar{c}_{a}^{\prime}<\bar{C}_{a}^{\prime \prime}$. The precise solution depends entirely on parameter values.

Case $\bar{p}_{e 0}<c_{r}<\bar{p}_{e \infty}$

Since demand is increasing and hence $\bar{p}_{e t}$ is increasing, the time path of $\bar{p}_{e t}$ may cut $c_{r}$ from below. We then get a pattern similar to the one illustrated in fig. 2 for the $S D$ case. It is sufficient that the $\bar{p}_{e t}$ curve be higher than the $\hat{p}_{t}$ curve over the interval $[0, \delta)$, higher than the $\breve{p}_{t}$ curve over the interval $[\delta, \breve{\theta})$ and lower than $c_{r}$ over some interval $[0, \delta)$ with $\delta<\breve{\theta}$. We may also obtain the sequence discussed in the preceding case $\bar{p}_{e \infty}<c_{r}$, provided that the time $\eta$ at which $\bar{p}_{e t}=c_{r}$ be later than the time $\tilde{\theta}$ at which $\tilde{p}_{t}=c_{r}$ (See figs. 3 and 4).

The solutions that are truly unique to the present case are illustrated in figs. 5, 6 and 7. They differ in terms of the location of the abatement period within the interval when the ceiling is binding. In the case of fig. 5 , the pollution stock increases to $Z_{\delta}=\bar{Z}$ during the first phase. At time $\delta, \hat{p}_{t}=\breve{p}_{t}$ and the ceiling binds with active abatement given by $a_{t}=\zeta\left[\breve{x}_{t}-\bar{x}\right]$. At time $\widetilde{\tau}$, $\breve{p}_{t}=\bar{p}_{e t}$ and abatement becomes too costly. During the next phase at the ceiling without abatement $[\widetilde{\tau}, \eta)$, only coal is used at its maximum limit and its price increases over time. At the beginning of the next phase $[\eta, \tilde{\theta})$, solar energy becomes economical, the ceiling is still binding and the price is equal to $c_{r}<\bar{p}_{e t}$, so that energy demand is more than what coal can provide, or $q_{t}=y_{c t}>\bar{x}$. Since coal is the cheaper resource, it is used at the maximum level $\bar{x}$ and solar supplies the residual, $y_{c t}-\bar{x}$. The use of solar power increases during this phase since energy 
demand continues to increase. At $\tilde{\theta}, \tilde{p}_{t}=c_{r}$ and coal ceases to be competitive. So it must be exhausted at this instant, with a corresponding jump in solar energy use to ensure continuity of the price path.

[Fig. 5 here]

In fig. 6 the solution is similar to the last one except that here the phase with active abatement is sandwiched between two phases during which no abatement takes place.

[Fig. 6 here]

Yet another pattern is shown in fig. 7, in which the abatement period occurs at the end of the ceiling and right before the deployment of solar energy. During a first phase $[\hat{\tau}, \tilde{\tau})$ at the ceiling, only coal is used. This is followed by active abatement during the interval $[\breve{\tau}, \breve{\theta})$ which in turn is followed by a phase $[\breve{\theta}, \tilde{\theta})$ in which both resources are consumed. At time $\breve{\theta}$ the extraction rate of coal falls from $y_{c \bar{\theta}}$ to $\bar{x}$, the number of units of pollution abated declines from $a_{\breve{\theta}}=\zeta\left(y_{c \breve{\theta}}-\bar{x}\right)$ to 0 and use of solar energy jumps from 0 to $y_{c \breve{\theta}}-\bar{x}$. Solar energy partly substitutes for coal and abatement activity. Coal use is constant during the period $[\breve{\theta}, \tilde{\theta})$ until it is exhausted at time $\tilde{\theta}$. Since the cost of coal under abatement rises with time, this case of an abatement period at the end of the ceiling can only arise in the increasing demand case. It can not occur under stationary demand, or as we shall see below, for decreasing demand.

[Fig. 7 here]

Case $\bar{p}_{e 0}>c_{r}$

Since $\bar{p}_{e t}$ increases with time, it may be above the cost of solar energy everywhere. The solution 
is analogous to that of $\bar{p}_{e}>c_{r}$ under stationary demand. ${ }^{14}$

\section{Decreasing Demand for Energy in the Long Run}

In the long run energy demand may decrease over time if global population declines due to lower fertility rates and energy consumption per capita reaches a plateau. Recent population projections suggest this trend (Lutz, Sanderson and Scherbov, 2001). ${ }^{15}$ In terms of our framework, falling demand implies that $\bar{p}_{e t}$ is decreasing and convex. Again, it is convenient to classify cases depending upon whether $\bar{p}_{e t}$ is always higher or lower than the cost of solar energy $c_{r}$, or cuts it from above. Since the first case ( $\bar{p}_{e t}$ is higher than $c_{r}$ ) is similar to the case last discussed, we focus on the remaining two: $\bar{p}_{e 0}<c_{r}$ and $\bar{p}_{e 0}>c_{r}>\bar{p}_{e \infty}$.

Case $\bar{p}_{e 0}<c_{r}$

When the unit abatement cost is sufficiently low, the extraction pattern is shown in fig. 8. It is exactly the same as in the stationary model with $\bar{p}_{e}<c_{r}$, except that when abatement does not occur, the price of energy at the ceiling is not constant but decreasing, since $\bar{p}_{e t}$ is decreasing exogenously. Coal extraction in this phase is constant at the maximum level $\bar{x}$. Thus the price of coal is increasing in the first two phases, then decreasing and increasing again during the Hotelling phase. It is clear from this figure that in general, when demand is decreasing abatement must be done at the beginning of the ceiling, and not in the strict interior or at the end, as we saw under increasing demand. Intuitively, the cost of coal under abatement rises over time, and with demand declining exogenously, it is valuable to abate earlier in the ceiling period if at all, and not later.

\footnotetext{
${ }^{14}$ except that $y_{c t}$ replaces $y_{c}$ in equations (A14) and (A15) in Appendix A.This case is also similar to $\bar{p}_{e \infty}>C_{r}$, i.e., $\bar{p}_{e t}>c_{r}, t \geq 0$ under decreasing demand, which is discussed below.

${ }^{15}$ World population is expected to rise from the current 6 billion to 9 billion in 2070 then decline to 8.4 billion in 2100. Fertility rates are falling below replacement levels not only in the developed countries but in some 71 intermediate-fertility developing countries. Energy demand is likely to follow this declining trend, albeit with a time lag that accounts for the near-term increase in energy consumption per capita in the developing countries.
} 
[Fig. 8 here]

Case $\bar{p}_{e 0}>c_{r}>\bar{p}_{e \infty}$

If demand were decreasing slowly, so that the time $\eta$ at which the $\bar{p}_{e t}$ curve cuts $c_{r}$ beyond time $\tilde{\theta}$ (see fig. 9), then the sequence of extraction will be exactly as in the stationary demand case of fig. 2. If demand for energy were to be moderately high initially and then decline rapidly then too we may obtain a trajectory similar to that in fig. 2 . More specifically the $\hat{p}_{e t}$ curve may cross $c_{r}$ before time $\breve{\theta}$ (see fig. 9). ${ }^{16}$

Finally when energy demand is sufficiently high but declining rapidly, we may obtain the unique extraction sequence shown in fig. 9. At the ceiling, pollution is abated initially during the interval $[\delta, \breve{\theta})$. At time $\breve{\theta}$, abatement stops and is replaced by use of solar energy during $[\breve{\theta}, \eta)$. In this time period, coal is used at the maximum level $\bar{x}$ supplemented by solar energy. However because the demand curve is shifting exogenously, overall energy consumption is declining and hence the residual use of solar $y_{c t}$ is decreasing. At the end of the phase $\bar{p}_{e t}=c_{r}$ and a new phase $[\eta, \tilde{\tau})$ begins in which $\bar{p}_{e t}$ is decreasing so that solar energy becomes too expensive and is no longer used, i.e., $p_{t}<c_{r}$. The price of energy at the ceiling decreases, and coal use is maintained at the maximum allowable limit $\bar{x}$. This phase ends at time $\tilde{\tau}$ when the $\bar{p}_{e t}$ graph intersects the Hotelling path $\tilde{p}_{t}$. During the next period $[\tilde{\tau}, \tilde{\theta})$, the ceiling constraint is no longer binding and extraction is according to Hotelling. Coal price increases until it is exhausted at time $\tilde{\theta}$ to be replaced by use of the backstop solar energy. What is unique about this extraction sequence is that solar energy is used during two disjoint time periods. When demand is high, solar is used at the ceiling to supplement coal use, but when demand declines, this expensive backstop is no longer necessary. All energy is supplied by coal, until it is exhausted and solar energy is deployed

\footnotetext{
${ }^{16}$ Another way to see this is from fig. 8. Imagine that $\bar{p}_{e 0}>c_{r}$ but that the $\hat{p}_{e t}$ curve is crossing the $c_{r}$ line at a time prior to $\hat{\tau}$.
} 
a second time in the terminal phase.

[Fig. 9 here]

\section{Concluding Remarks}

This paper is a first attempt at extending Hotelling theory to the case when the stock of pollution from using a nonrenewable resource is constrained. The pollution stock has an upper bound, possibly the result of an international agreement. As in standard Hotelling models, an expensive backstop such as solar energy can provide clean energy. The question is: what is the sequence of extraction of coal and solar energy given the possibility of costly abatement and exogenously changing demand for energy? We consider the simple but restrictive case of stationary demand, and two likely scenarios: increasing demand for energy in the short run and falling demand in the long run.

One general result is that in all cases, coal is used exclusively in the initial period and the stock of pollution builds up over time, followed by an interval in which the ceiling is binding. Beyond this interval, the emission stock declines to zero as energy supply shifts from the exclusive use of the nonrenewable to that of the backstop resource. However, the details of this transition differ markedly from case to case. If solar energy is cheap or coal is highly polluting, or the imposed ceiling is low, both resources may be used exactly at the instant the ceiling is attained. This pattern is maintained until the nonrenewable resource is exhausted. Alternatively when coal is abundant or mildly polluting, or solar energy is expensive, the supply of energy at the ceiling is provided only by coal, followed by a Hotelling phase when the ceiling is non-binding in which extraction declines until coal is exhausted and is replaced by the backstop resource. A unique pattern is obtained when energy demand is decreasing. Both resources may be used at the ceiling, followed by the exclusive use of coal, and finally exclusive use of the backstop in the terminal period. It suggests two disjoint periods of time when the clean backstop may be used.

When emissions can be abated at constant cost, such as through carbon sequestration by forests, abatement does not occur if its cost is sufficiently high. If the cost is sufficiently low, abatement is only done when the stock is at the ceiling. Under stationary and decreasing demand, abatement 
must only be done at the beginning of the ceiling period. However, when demand is increasing, as the unit cost of abatement rises, abatement may be done at the beginning of the ceiling period, strictly in the interior or at the very end. Abatement competes with the clean backstop resource and only one or the other is employed at any given time.

Useful policy insights can be obtained even from the stylized model developed here. One implication is that the standard Hotelling solution of a transition from a polluting fossil fuel to a clean renewable resource may be overly simplistic when there is a ceiling on the stock of emissions. There may be a strong case for use of the renewable resource during the period when the ceiling is tight, even though the cost of the backstop is higher than that of the fossil fuel and the latter has not been exhausted. Thus, solar or other renewable technologies may need to be used to supplement the use of fossil fuel resources, even if they are not economical in terms of the unit cost of energy. ${ }^{17}$ Since coal is relatively more abundant than oil and gas, and it is realistic to expect global energy demand to peak and then decline over the long-term, the joint extraction of fossil fuels and solar energy may be feasible under an international agreement. For instance, in order to achieve compliance with environmental targets mandated by the Kyoto Treaty, we may use expensive solar energy for a time when the ceiling is binding. Then as energy demand declines over time, abandon solar and revert back to a "Hotelling" world with coal as the primary source of energy, only to transition completely to solar energy when coal is exhausted. Empirical work needs to be done using this Hotelling framework to see which of the cases considered in the paper are likely given plausible parameter values.

Modeling a nonrenewable resource with a pollution ceiling is a first step towards developing theory that can examine substitution across multiple energy resources (oil, coal, natural gas and renewables) under alternative environmental targets. Empirical trends such as the recent transition from coal burning to the cleaner natural gas in power generation can be better examined in a model with multiple nonrenewable resources each with different emission characteristics. For example, one can study the optimal extraction sequence with two scarce

\footnotetext{
${ }^{17}$ Conventional wisdom, which suggests that renewables cannot be used because they are currently costlier than fossil fuels, may be somewhat misplaced.
} 
resources - a cheap polluting resource (e.g., coal) and a costly clean resource (e.g., natural gas). In future work, one could specify nonlinear abatement (e.g., the unit cost of bringing land under forests may rise with volume abated) and cost structures for the backstop resource, in which case both these options may be used simultaneously at the ceiling. The cost of the clean backstop itself may be the outcome of a research and development process. Yet another possible extension could include the endogenous choice of a ceiling and its welfare and political economy implications. It may be realistic to expect the ceiling to become more stringent over time as societies become more sensitive to environmental externalities. In a model with multiple countries, the choice of a ceiling may be the outcome of an explicit bargaining process. 


\section{Appendix A}

Stationary Demand: $\bar{p}_{e}<C_{r}$

When abatement costs are sufficiently low, there are six unknowns: $\lambda_{0}, \mu_{0}, \delta, \tilde{\tau}, \tilde{\tau}$ and $\tilde{\theta}$, with six equations as follows:

- $\quad$ the supply/cumulative demand balance for coal:

$$
\int_{0}^{\delta} \hat{x}_{z}\left(\lambda_{0}, \mu_{0}\right) d z+\int_{\delta}^{\breve{\tau}} \breve{X}_{z}\left(\lambda_{0}\right) d z+[\tilde{\tau}-\widetilde{\tau}] \bar{x}+\int_{\tilde{\tau}}^{\tilde{\theta}} \tilde{x}_{z}\left(\lambda_{0}\right) d z=X_{0}
$$

- $\quad$ continuity of the pollution stock at the ceiling:

$$
\hat{Z}_{\delta}\left(\lambda_{0}, \mu_{0}\right)=\bar{Z}
$$

- $\quad$ and continuity of the price path:

$$
\hat{p}_{\delta}\left(\lambda_{0}, \mu_{0}\right)=p_{\delta}\left(\lambda_{0}\right), \quad \breve{p}_{\widetilde{\tau}}\left(\lambda_{0}\right)=\bar{p}_{e}, \quad \tilde{p}_{\tilde{\tau}}\left(\lambda_{0}\right)=\bar{p}_{e}, \quad \text { and } \quad \tilde{p}_{\tilde{\theta}}\left(\lambda_{0}\right)=c_{r} \text {. }
$$

When abatement costs are high, the problem will have five unknowns, $\lambda_{0}, \mu_{0}, \hat{\tau}, \tilde{\tau}$ and $\tilde{\theta}$, which solve the following five equations system:

- $\quad$ the supply/cumulative demand balance for coal:

$$
\int_{0}^{\hat{\tau}} \hat{x}_{z}\left(\lambda_{0}, \mu_{0}\right) d z+[\tilde{\tau}-\bar{\tau}] \bar{X}+\int_{\tilde{\tau}}^{\tilde{\theta}} \tilde{X}_{z}\left(\lambda_{0}\right) d z=X_{0}
$$

- $\quad$ continuity of the pollution stock at the ceiling:

$$
\hat{Z}_{\hat{\tau}}\left(\lambda_{0}, \mu_{0}\right)=\bar{Z}
$$

- $\quad$ and continuity of the price path:

$$
\hat{p}_{\hat{\tau}}\left(\lambda_{0}, \mu_{0}\right)=\bar{p}_{e}, \quad \tilde{p}_{\tilde{\tau}}\left(\lambda_{0}\right)=\bar{p}_{e}, \quad \text { and } \quad \tilde{p}_{\tilde{\theta}}\left(\lambda_{0}\right)=c_{r}
$$

How large does the abatement cost $c_{a}$ have to be for no abatement to occur? First we solve the problem without abatement. Let $\lambda_{0}^{*}, \mu_{0}^{*}, \hat{\theta}^{*}, \widetilde{\tau}^{*}$ and $\widetilde{\theta}^{*}$ be the solution. Next consider $\breve{p}_{t}^{*}$ defined as the solution to $\breve{p}_{t}^{*}=c_{e}+\lambda_{0}^{*} e^{\rho t}+\zeta C_{a}$. Then either $\breve{p}_{t}^{*} \geq \hat{p}_{t}, t \in\left[0, \hat{\theta}^{*}\right)$, in which case the solution has no abatement, or there 
exists some subinterval of $\left[0, \hat{\theta}^{*}\right)$ during which $\breve{p}_{t}^{*}<\hat{p}_{t}^{*}$ and abatement occurs. There exists a critical value of the abatement cost $\bar{C}_{a}$, with abatement always occurring at lower values of $c_{a}$ and no abatement at higher values. In Appendix B we show that if there exist $\lambda_{0}, \mu_{0}, \delta, \tilde{\tau}, \tilde{\tau}$ and $\tilde{\theta}$ satisfying (A1)-(A3) or $\lambda_{0}, \mu_{0}, \hat{\tau}, \tilde{\tau}$ and $\tilde{\theta}$ satisfying (A4)-(A6), all the necessary conditions (2)-(12) are satisfied. We summarize:

Proposition 1: For $S D$ with $\bar{p}_{e}<C_{r}$, there exists a critical value $\bar{c}_{a}$ of the abatement cost such that:

a. either $C_{a} \geq \bar{c}_{a}$ and the optimal path has four phases with $\lambda_{0}, \mu_{0}, \hat{\tau}, \tilde{\tau}$ and $\tilde{\theta}$ satisfying (A4)-(A6) and

$$
x_{t}=\left\{\begin{array}{ll}
\hat{x}_{t}, & t \in[0, \hat{\tau}) \\
\bar{x} & , \quad t \in[\hat{\tau}, \tilde{\tau}) \\
\tilde{x}_{t}, & t \in[\tilde{\tau}, \tilde{\theta}) \\
0, & t \in[\tilde{\theta}, \infty)
\end{array}, \quad y_{t}=\left\{\begin{array}{ccc}
0, & t \in[0, \tilde{\theta}) \\
y_{c}, & t \in[\tilde{\theta}, \infty)
\end{array}, \quad a_{t}=0, t \in[0, \infty),\right.\right.
$$

b. or $C_{a}<\bar{C}_{a}$ and the optimal path has five phases with characteristics $\lambda_{0}, \mu_{0}, \delta, \widetilde{\tau}, \tilde{\tau}$ and $\tilde{\theta}$ satisfying (A4)-(A6) and

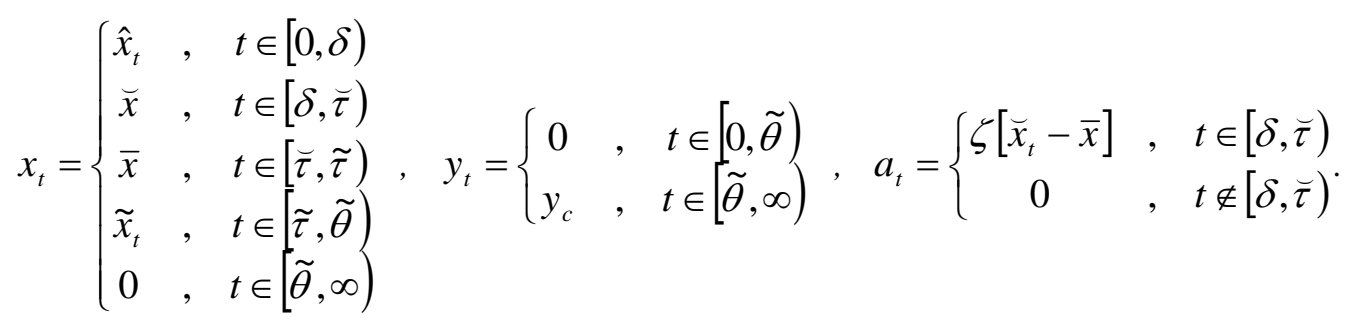

Stationary Demand: $\bar{p}_{e}>c_{r}$

With active abatement, there are five unknowns $\lambda_{0}, \mu_{0}, \delta, \breve{\theta}$ and $\tilde{\theta}$, and five equations:

- the supply/cumulative demand balance for coal:

$$
\int_{0}^{\delta} \hat{x}_{z}\left(\lambda_{0}, \mu_{0}\right) d z+\int_{\delta}^{\breve{\theta}} \breve{x}_{z}\left(\lambda_{0}, \mu_{0}\right) d z+[\tilde{\theta}-\breve{\theta}] \bar{x}=X_{0}
$$

- continuity of the pollution stock at the ceiling, identical to (A2),

- $\quad$ and continuity of the price path: 


$$
\hat{p}_{\delta}\left(\lambda_{0}, \mu_{0}\right)=\breve{p}_{t}\left(\lambda_{0}\right), \quad \breve{p}_{\breve{\theta}}\left(\lambda_{0}\right)=c_{r} \text {. and } \quad \tilde{p}_{\tilde{\theta}}\left(\lambda_{0}\right)=c_{r} \text {. }
$$

As before, when $c_{a}$ is large enough, the $\breve{p}_{t}$ curve lies above the $\hat{p}_{t}$ curve over the time interval $[0, \hat{\theta}$ ) (see fig. 2). The abatement phase will disappear. The optimal sequence involves four unknowns $\lambda_{0}, \mu_{0}, \hat{\theta}$ and $\tilde{\theta}$ and four equations:

- $\quad$ the supply/cumulative demand balance for coal:

$$
\int_{0}^{\hat{\theta}} \hat{x}_{z}\left(\lambda_{0}, \mu_{0}\right) d z+[\tilde{\theta}-\hat{\theta}] \bar{x}=X_{0}
$$

- $\quad$ continuity of the pollution stock at the ceiling, $\hat{Z}_{\hat{\theta}}\left(\lambda_{0}, \mu_{0}\right)=\bar{Z}$,

- $\quad$ and continuity of the price path:

$$
\hat{p}_{\hat{\theta}}\left(\lambda_{0}, \mu_{0}\right)=c_{r} \text { and } \tilde{p}_{\tilde{\theta}}\left(\lambda_{0}\right)=c_{r} .
$$

Whether abatement occurs can be determined as before. Let $\lambda_{0}^{*}, \mu_{0}^{*}, \hat{\theta}^{*}$ and $\tilde{\theta}^{*}$ be the solution to the problem absent active abatement and let $\breve{p}_{t}^{*}=c_{e}+\lambda_{0}^{*} e^{\rho t}+\zeta c_{a}$. Then either $\breve{p}_{t}^{*} \geq \hat{p}_{t}^{*}, t \in\left[0, \hat{\theta}^{*}\right)$ and it is optimal not to abate or there exists some subinterval of $\left[0, \hat{\theta}^{*}\right)$ during which $\breve{p}_{t}^{*}<\hat{p}_{t}^{*}$ and active abatement occurs. Clearly there exists a critical abatement cost $\bar{C}_{a}$ such that there is no abatement if $c_{a} \geq \bar{C}_{a}$ and active abatement if $c_{a}<\bar{C}_{a}$. We can summarize:

Proposition 2: For $S D$ with $\bar{p}_{e}>c_{r}$, there exists a critical value $\bar{c}_{a}$ of the abatement cost such that:

a. $\quad$ either $C_{a} \geq \bar{C}_{a}$ and the solution has three phases with $\lambda_{0}, \mu_{0}, \hat{\theta}$ and $\tilde{\theta}$ satisfying (A11)-(A13) and

$$
x_{t}=\left\{\begin{array}{ccc}
\hat{x}_{t}, & t \in[0, \hat{\theta}) \\
\bar{x} & , & t \in[\hat{\theta}, \tilde{\theta}) \\
0, & t \in[\tilde{\theta}, \infty)
\end{array}\right), \quad y_{t}=\left\{\begin{array}{ccc}
0, & t \in[0, \hat{\theta}) \\
y_{c}-\bar{x}, & t \in[\hat{\theta}, \tilde{\theta}) \\
y_{c}, & t \in[\tilde{\theta}, \infty]
\end{array}, \quad a_{t}=0, t \in[0, \infty)\right. \text {, }
$$


b. or $C_{a}<\bar{C}_{a}$ with a solution with four phases with $\lambda_{0}, \mu_{0}, \delta, \breve{\theta}$ and $\tilde{\theta}$ satisfying (A2),(A9) and (A10) and

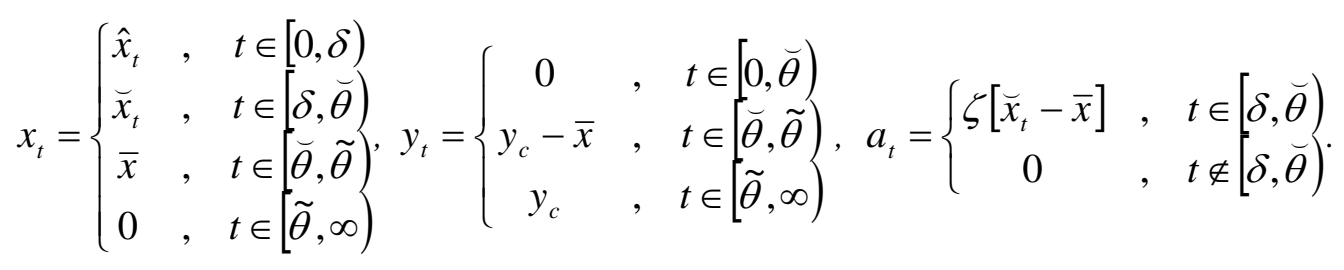

Increasing Demand: $\bar{p}_{e \infty}<c_{r}$

For the case shown in fig. $3, \lambda_{0}, \mu_{0}, \delta, \widetilde{\tau}, \tilde{\tau}$ and $\tilde{\theta}$ are the solution of a system of equations similar to (A1)-(A3) and the extraction sequence is determined by (A8) (Proposition 1b) with $y_{c t}$ instead of $y_{c}$.

For fig. 4, the sequence has six phases: $p_{t}=\hat{p}_{t}$ and $q_{t}=\hat{x}_{t}>\bar{x}$ for $t \in[0, \delta), p_{t}=\bar{p}_{e t}$ and $q_{t}=x_{t}=\bar{x}$ for $t \in\left[\delta, \breve{\tau}_{1}\right) \cup\left[\breve{\tau}_{2}, \tilde{\tau}\right), p_{t}=\breve{p}_{t}, q_{t}=x_{t}=\breve{x}_{t}>\bar{x}$ and $a_{t}=\zeta\left[\breve{x}_{t}-\bar{x}\right]$ for $t \in\left[\breve{\tau}_{1}, \breve{\tau}_{2}\right), p_{t}=\tilde{p}_{t}$ and $q_{t}=\tilde{q}_{t}$ for $t \in[\tilde{\tau}, \tilde{\theta})$, and lastly $p_{t}=c_{r}$ and $q_{t}=y_{t}=y_{c t}$ for $t \in[\tilde{\theta}, \infty)$. The seven characteristics of the sequence, $\lambda_{0}, \mu_{0}, \delta, \breve{\tau}_{1}, \breve{\tau}_{2}, \tilde{\tau}$ and $\tilde{\theta}$ are the solution to the following system of seven equations:

- the supply/cumulative demand balance for coal:

$$
\int_{0}^{\delta} \hat{x}_{z}\left(\lambda_{0}, \mu_{0}\right) d z+\left[\left(\breve{\tau}_{1}-\delta\right)+\left(\tilde{\tau}-\breve{\tau}_{2}\right)\right] \bar{x}+\int_{\widetilde{\tau}_{1}}^{\breve{\tau}_{2}} \breve{x}_{z}\left(\lambda_{0}, \mu_{0}\right) d z+\int_{\tilde{\tau}}^{\tilde{\theta}} \tilde{x}_{z}\left(\lambda_{0}\right) d z=X_{0}
$$

- the continuity of the pollution stock at time $\delta$ given by (A2),

- $\quad$ and continuity of the price path: $:^{18}$

$$
\hat{p}_{\delta}\left(\lambda_{0}, \mu_{0}\right)=\bar{p}_{e \delta}, \quad \bar{p}_{e \bar{\tau}_{1}}=\breve{p}_{\check{\tau}_{1}}, \quad \bar{p}_{e \breve{\tau}_{2}}=\breve{p}_{\bar{\tau}_{2}} \bar{p}_{e \tilde{\tau}}=\tilde{p}_{\tilde{\tau}} \text {, and } \quad \tilde{p}_{\tilde{\theta}}=c_{r}
$$

For sufficiently high abatement costs the curve $\breve{p}_{t}$ is located above $\hat{p}_{t}$ over the time interval $[0, \hat{\tau})$ during which $\bar{p}_{e t}>\hat{p}_{t}$, and above the curve $\bar{p}_{e t}$ over the time interval $[\hat{\tau}, \tilde{\tau})$. Hence the phase during which it would be optimal

\footnotetext{
${ }^{18}$ Since $\bar{p}_{e t}$ is concave and $\breve{p}_{t}$ is convex, they may cross at most twice.
} 
to abate disappears and the optimal sequence of resource use has four phases: $p_{t}=\hat{p}_{t}, q_{t}=x_{t}=\bar{x}$ and $Z_{t}=\hat{Z}_{t}$ for $t \in[0, \tilde{\tau}), p_{t}=\bar{p}_{e t}, q_{t}=x_{t}=\bar{x}$ and $Z_{t}=\bar{Z}_{t}$ for $t \in[\hat{\tau}, \tilde{\tau}), p_{t}=\tilde{p}_{t}$ and $q_{t}=x_{t}=\tilde{x}_{t}$ for $t \in[\tilde{\tau}, \tilde{\theta})$, and $p_{t}=c_{r}$ and $q_{t}=y_{t}=y_{c t}$ for $t \in[\tilde{\theta}, \infty)$. The values of the five variables characterizing the sequence $\lambda_{0}, \mu_{0}, \hat{\tau}, \tilde{\tau}$ and $\tilde{\theta}$ are the solution to the following system of five equations:

- the supply/cumulative demand balance for coal which is formally identical to equation (A4),

- continuity of the pollution stock given by (A5) since the ceiling is attained at $\hat{\tau}$,

- $\quad$ and continuity of the price path given by (A6) with $\bar{p}_{e t}$ instead of $\bar{p}_{e}$ :

$$
\hat{p}_{\hat{\tau}}\left(\lambda_{0}, \mu_{0}\right)=\bar{p}_{e \hat{\tau}}, \quad \tilde{p}_{\tilde{\tau}}\left(\lambda_{0}\right)=\bar{p}_{e \tilde{\tau}} \quad \text { and } \quad \tilde{p}_{\tilde{\theta}}\left(\lambda_{0}\right)=c_{r} .
$$

We can state

Proposition 3: For ID with $\bar{p}_{e \infty}<C_{r}$ :

a. either there exists a critical value of the abatement cost $\bar{C}_{a}$, such that:

a.1 for $c_{a} \geq \bar{c}_{a}$ the optimal path has four phases with $\lambda_{0}, \mu_{0}, \hat{\tau}, \tilde{\tau}$ and $\tilde{\theta}$, satisfying (A4), (A5) and (A8), the optimal policy being given by (A7) with $y_{c t}$ instead of $y_{c}$, a.2 for $\bar{c}_{a}>c_{a}$ the optimal path has five phases with $\lambda_{0}, \mu_{0}, \delta, \tilde{\tau}, \tilde{\tau}$ and $\tilde{\theta}$, satisfying (A2), (A16) and (A17), the optimal policy being given by (A8) with $y_{c t}$ instead of $y_{c}$,

b. or there exist two critical values of the abatement cost, $\bar{C}_{a}^{\prime}$ and $\bar{C}_{a}^{\prime \prime}$, with $\bar{C}_{a}^{\prime}<\bar{C}_{a}^{\prime \prime}$, such that:

b.1 for $C_{a} \geq \bar{c}_{a}$ the optimal path is the pattern characterized in a.1,

b.2 for $\bar{C}_{a}^{\prime \prime}>c_{a} \geq \bar{c}_{a}^{\prime}$, the optimal path has six phases with characteristics $\lambda_{0}, \mu_{0}, \delta, \widetilde{\tau}_{1}, \breve{\tau}_{2}, \tilde{\tau}$ and $\tilde{\theta}$ satisfying (A2), (A16) and (A17), the optimal policy being given by: 


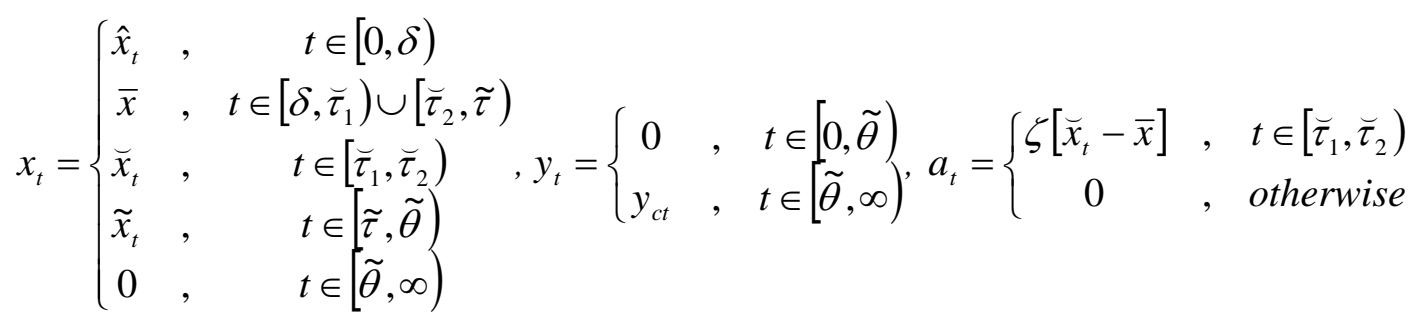

b.3 for $\bar{C}_{a}^{\prime}>c_{a}$ the optimal pattern is the pattern characterized in a.2.

Increasing Demand: $\bar{p}_{e 0}<c_{r}<\bar{p}_{e \infty}$

This five phase sequence (see fig. 5) is characterized by the values of the six variables $\lambda_{0}, \mu_{0}, \delta, \widetilde{\tau}, \eta$ and $\tilde{\theta}$, solution to the following system of six equations:

- the supply/cumulative demand balance for coal:

$$
\int_{0}^{\delta} \hat{x}_{z}\left(\lambda_{0}, \mu_{0}\right) d z+[\tilde{\theta}-\delta] \bar{x}=X_{0},
$$

- $\quad$ continuity of the pollution stock at $\delta$ given by (A2),

- $\quad$ and the price path continuity equations:

$$
\hat{p}_{\delta}\left(\lambda_{0}, \mu_{0}\right)=\breve{p}_{\delta}\left(\lambda_{0}\right), \quad \breve{p}_{\breve{\tau}}\left(\lambda_{0}\right)=\bar{p}_{e \tilde{\tau}}, \quad \bar{p}_{e \eta}=c_{r} \quad \text { and } \quad \tilde{p}_{\tilde{\theta}}\left(\lambda_{0}\right)=c_{r} .
$$

For fig. 6, the sequence is characterized by the values of the seven variables $\lambda_{0}, \mu_{0}, \hat{\tau}, \breve{\tau}_{1}, \breve{\tau}_{2} \eta$ and $\tilde{\theta}$, solution of the following system of seven equations:

- the supply/cumulative demand balance for coal:

$$
\int_{0}^{\hat{\imath}} \hat{x}_{z}\left(\lambda_{0}, \mu_{0}\right) d z+[\tilde{\theta}-\hat{\tau}] \bar{x}=X_{0}
$$

- continuity of the pollution stock at $\hat{\tau}$ given by (A5),

- $\quad$ and the price path continuity equations: 


$$
\hat{p}_{\hat{\tau}}\left(\lambda_{0}, \mu_{0}\right)=\bar{p}_{e \hat{\tau}}, \quad \bar{p}_{e \breve{\tau}_{1}}=\breve{p}_{\breve{\tau}_{1}}\left(\lambda_{0}\right), \quad \bar{p}_{e \hat{\tau}_{2}}=\breve{p}_{\breve{\tau}_{2}}\left(\lambda_{0}\right), \quad \bar{p}_{e \eta}=c_{r} \quad \text { and } \quad \tilde{p}_{\tilde{\theta}}\left(\lambda_{0}\right)=c_{r}
$$

Finally for fig. 7, the five phase sequence is characterized by the values of the six variables $\lambda_{0}, \mu_{0}, \hat{\tau}, \breve{\tau}, \breve{\theta}$ and $\tilde{\theta}$, solution of the following system of six equations:

- $\quad$ the supply/cumulative demand balance for coal identical to (A22),

- $\quad$ continuity of the pollution stock equation at $\hat{\tau}$, that is equation (A5), and

- and the price path continuity equations:

$$
\hat{p}_{\hat{\tau}}\left(\lambda_{0}, \mu_{0}\right)=\bar{p}_{e \hat{\tau}}, \quad \bar{p}_{e \breve{\tau}}=\breve{p}_{\breve{\tau}}, \quad \breve{p}_{e \breve{\theta}}=c_{r} \quad \text { and } \quad \tilde{p}_{\tilde{\theta}}\left(\lambda_{0}\right)=c_{r} .
$$

Several patterns may emerge as $C_{a}$ increases from zero, the free abatement case, to the other extreme of a high $C_{a}$ in which there is no abatement. Proposition 4 summarizes the discussion.

Proposition 4: For ID with $\bar{p}_{e 0}<c_{r}<\bar{p}_{e \infty}$, we may have the following sequence of phases:

a. the same pattern as in SD characterized in Proposition 2,

b. the same pattern as in ID with $\bar{p}_{e \infty}<c_{r}$ characterized in Proposition 3,

c. the patterns illustrated in Figures 5,6 and 7, which are, respectively:

c.1 The five phase sequence with characteristics $\lambda_{0}, \mu_{0}, \delta, \widetilde{\tau}, \eta$ and $\tilde{\theta}$, satisfying the equations (A2), (A20) and (A21), the optimal policy being given by

$$
x_{t}=\left\{\begin{array}{lll}
\hat{x}_{t}, & t \in[0, \delta) \\
\widetilde{x}_{t}, & t \in[\delta, \widetilde{\tau}) \\
\bar{x}, & t \in[\widetilde{\tau}, \tilde{\theta}) \\
0, & t \in[\tilde{\theta}, \infty)
\end{array}, y_{t}=\left\{\begin{array}{cl}
0, & t \in[0, \eta) \\
y_{c t}-\bar{x}, & t \in[\eta, \widetilde{\theta}) \\
y_{c t}, & t \in[\tilde{\theta}, \infty)
\end{array}, a_{t}=\left\{\begin{array}{cc}
\zeta\left[\breve{x}_{t}-\bar{x}\right], & t \in[\delta, \widetilde{\tau}) \\
0, & \text { otherwise }
\end{array}\right.\right.\right.
$$

c.2 The six phase pattern with $\lambda_{0}, \mu_{0}, \hat{\tau}, \breve{\tau}_{1}, \breve{\tau}_{2} \eta$ and $\tilde{\theta}$, satisfying the equations (A5), (A22) and (A23), the optimal policy being given by 


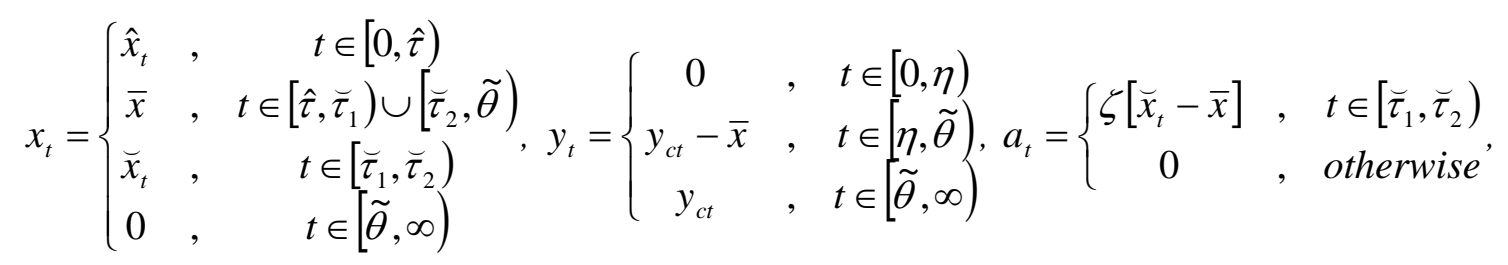

and

c.3 The five phase sequence with $\lambda_{0}, \mu_{0}, \hat{\tau}, \breve{\tau}, \breve{\theta}$ and $\tilde{\theta}$, satisfying the equations (A5), (A22) and (A24), the optimal policy being given by

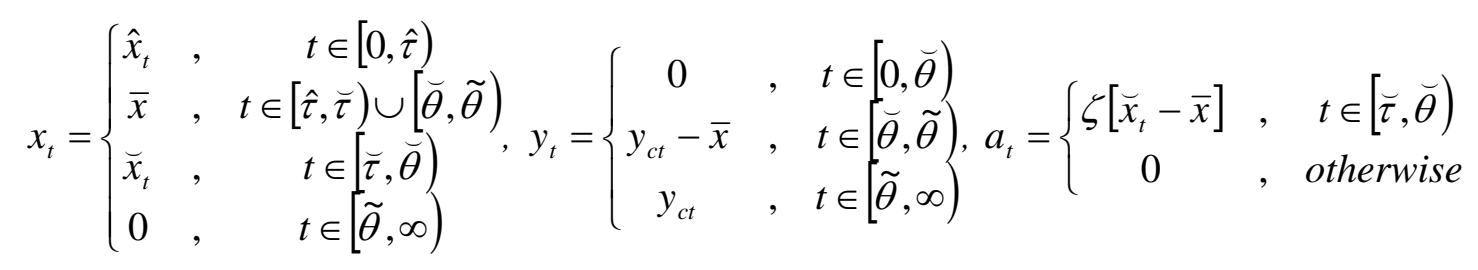

Increasing Demand: $\bar{p}_{e 0}>C_{r}$

Proposition 5: For ID with $\bar{p}_{e 0}>c_{r}$ and for DD with $\bar{p}_{e \infty}>c_{r}$ the optimal sequence is the same as in Proposition 2 with $y_{c t}$ instead of $y_{c}$ in equations (A8) and (A9).

Decreasing Demand: $\bar{p}_{e 0}<c_{r}$

Proposition 6: For $D D$ with $\bar{p}_{e 0}<c_{r}$, the optimal sequence is the same as in Proposition 1 with $\bar{p}_{e t}$ and $y_{c t}$ substituted for $\bar{p}_{e}$ and $y_{c}$, respectively.

Decreasing Demand: $\bar{p}_{e 0}>c_{r}>\bar{p}_{e \infty}$

The sequence is characterized by the values of the seven variables $\lambda_{0}, \mu_{0}, \delta, \breve{\theta}, \eta, \tilde{x}$ and $\tilde{\theta}$, the solution to the following system of seven equations:

- $\quad$ the supply/cumulative demand balance for coal: 


$$
\int_{0}^{\delta} \hat{x}_{z}\left(\lambda_{0}, \mu_{0}\right) d z+\int_{\delta}^{\breve{\theta}} \breve{x}_{z}\left(\lambda_{0}, \mu_{0}\right) d z+[\tilde{\tau}-\eta] \bar{x}+\int_{\tilde{\tau}}^{\tilde{\theta}} \tilde{x}_{z}\left(\lambda_{0}\right) d z=X_{0}
$$

- continuity of the pollution stock at time $\delta$ given by (A2),

- $\quad$ and the price path continuity equations:

$$
\hat{p}_{\delta}\left(\lambda_{0}, \mu_{0}\right)=\breve{p}_{\delta}\left(\lambda_{0}\right), \quad \breve{p}_{\breve{\theta}}=c_{r}, \quad \bar{p}_{e \eta}=c_{r}, \quad \tilde{p}_{\tilde{\tau}}\left(\lambda_{0}\right)=\bar{p}_{\tilde{\tau}} \quad \text { and } \quad \tilde{p}_{\tilde{\theta}}\left(\lambda_{0}\right)=c_{r} .
$$

Two different types of paths may be optimal for very low abatement costs. If abatement is costless, the price path would be pure Hotelling with as much abatement at any instant of time as necessary. Since there would be no restriction on the extraction rate, the rent must be higher than the optimal rent under a strictly positive abatement cost.

The optimal rent in the zero cost of abatement case may be $\lambda_{0}^{\prime}>\lambda_{0}$ (see fig. 9), generating the Hotelling price path $\tilde{p}_{t}^{\prime}=c_{e}+\lambda_{0}^{\prime} e^{\rho t}$, crossing the curve $\bar{p}_{e t}$ at a time at which $\bar{p}_{e t}=c_{r}$. Then for positive but very small abatement costs, the optimal price path is very close to the path $\tilde{p}_{t}^{\prime}$ with an extraction sequence as depicted in fig. 8 . For higher abatement costs we may obtain the sequence just described (equations (A2), (A28) and (A29)). But an even higher scarcity rent in the costless abatement case, say $\lambda_{0}^{\prime \prime}>\lambda_{0}^{\prime}$ may generate a path $\tilde{p}_{t}^{\prime \prime}=c_{e}+\lambda_{0}^{\prime \prime} e^{\rho t}$ which crosses the $\bar{p}_{e t}$ curve at a time when $\bar{p}_{e t}>c_{r}$ (see fig. 9). For positive but very low abatement costs, the optimal price path is now very close to the path $\tilde{p}_{t}^{\prime \prime}$, but of the type depicted in fig. 2, that is never decreasing. For higher abatement costs it is the price path described previously (equations (A2), (A28) and (A29))

If the abatement cost is very high, abatement is always too costly, hence the active abatement phase $[\delta, \breve{\theta})$

disappears because the curve $\breve{p}_{t}$ is located above the curve $\hat{p}_{t}$ over the time interval $[0, \hat{\theta})$ during which $\hat{p}_{t}<c_{r}$. Then the optimal sequence has five phases as shown in fig. 9. The six characteristics of the sequence, $\lambda_{0}, \mu_{0}, \hat{\theta}, \eta, \tilde{\tau}$ and $\tilde{\theta}$ are the solution of the following system of six equations: 
- $\quad$ the supply/cumulative demand balance for coal:

$$
\int_{0}^{\hat{\theta}} \hat{x}_{z}\left(\lambda_{0}, \mu_{0}\right) d z+[\tilde{\tau}-\hat{\theta}] \bar{x}+\int_{\tilde{\tau}}^{\tilde{\theta}} \tilde{x}_{z}\left(\lambda_{0}\right) d z=X_{0},
$$

- $\quad$ continuity of the pollution stock at time $\hat{\theta}$ given by (A12),

- $\quad$ and the price paths continuity equations:

$$
\hat{p}_{\hat{\theta}}\left(\lambda_{0}, \mu_{0}\right)=c_{r}, \quad \bar{p}_{e \eta}=c_{r}, \quad \bar{p}_{e \tilde{\tau}}=\tilde{p}_{\tilde{\tau}}\left(\lambda_{0}\right) \text {, and } \tilde{p}_{\tilde{\theta}}\left(\lambda_{0}\right)=c_{r} \text {. }
$$

\section{We summarize:}

Proposition 7: For DD with $\bar{p}_{e 0}>c_{r}>\bar{p}_{e \infty}$ :

a. $\quad$ either there exists a critical value $\bar{C}_{a}$ of the abatement cost such that, depending upon whether $C_{a} \geq \bar{C}_{a}$ or $c_{a}<\bar{C}_{a}$, we have the sequence described in Proposition 6,

b. $\quad$ or there exists a critical value $\bar{C}_{a}$ such that :

b.1 For $c_{a} \geq \bar{c}_{a}$, the optimal sequence has five phases with characteristics $\lambda_{0}, \mu_{0}, \hat{\theta}, \eta, \tilde{\tau}$ and $\tilde{\theta}$ satisfying (A12), (A30) and (A31), and the optimal policy is :

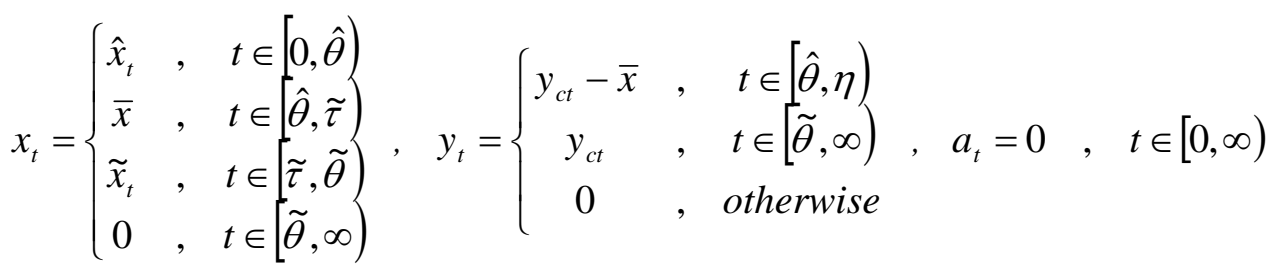

b.2 For $c_{a}<\bar{C}_{a}$, the optimal sequence has six phases with characteristics $\lambda_{0}, \mu_{0}, \delta, \breve{\theta}, \eta, \tilde{x}$ and $\tilde{\theta}$, satisfying (A2), (A28) and (A29), and the optimal policy is given by:

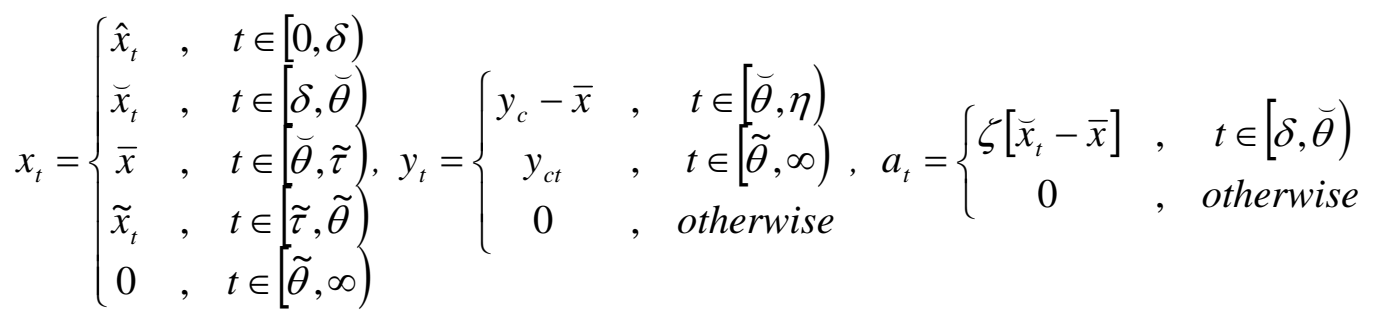


c. or lastly there exist two critical values of the abatement cost, $\bar{C}_{a}^{\prime}$ and $\bar{C}_{a}^{\prime \prime}, \bar{C}_{a}^{\prime}<\bar{C}_{a}^{\prime \prime}$, such that :

c.1 For $c_{a} \geq \bar{c}_{a}^{\prime \prime}$, the optimal sequence is the same as in b.1,

c.2 For $c_{a} \in\left(\bar{C}_{a}^{\prime}, \bar{C}_{a}^{\prime \prime}\right)$, the optimal sequences is the same as in b.2,

c.3 For $C_{a} \leq \bar{C}_{a}^{\prime}$, the optimal sequence is the same as in Proposition 2.b and (A15). 


\section{Appendix B}

B.1 Consider $S D$ with $\bar{p}_{e}<c_{r}$ and assume first that there exist $\lambda_{0}, \mu_{0}, \hat{\tau}, \tilde{\tau}$ and $\tilde{\theta}$ satisfying (A4)-(A6) and that the solution is given by (A7). It is easy to check that the non-negative functions $\gamma_{a t}, \gamma_{e t}, \gamma_{r t},-\mu_{t}$ and $v_{t}$ defined below are such that the equations (2)-(12) are satisfied. Since coal is exhausted at time $\tilde{\theta}$ the transversality condition (11) is satisfied. It implies that $\lim _{t \uparrow+\infty} Z_{t}=0$. Below we show that $\mu_{t}=0$ for $t \geq \tilde{\tau}$, so that the transversality condition (12) is satisfied.

$\gamma_{a t}= \begin{cases}c_{a}+\mu_{0} e^{(\rho+\alpha) t} & , \quad t \in[0, \hat{\tau}) \\ c_{a}-\zeta\left[\bar{p}_{e}-\tilde{p}_{t}\right] & , \quad t \in[\hat{\tau}, \tilde{\tau}) \\ c_{a} & , \quad t \in[\tilde{\tau}, \infty)\end{cases}$

$\gamma_{e t}= \begin{cases}0 & , \quad t \in[0, \tilde{\theta}) \\ \tilde{p}_{t}-c_{r} & , \quad t \in[\tilde{\theta}, \infty)\end{cases}$

$\gamma_{r t}=\left\{\begin{array}{lll}c_{r}-\hat{p}_{t} & , \quad t \in[0, \hat{\tau}) \\ c_{r}-\bar{p}_{e} & , \quad t \in[\hat{\tau}, \tilde{\tau}) \\ c_{r}-\breve{p}_{t} & , \quad t \in[\tilde{\tau}, \tilde{\theta}) \\ 0 & , \quad t \in[\tilde{\theta}, \infty)\end{array}\right.$

$\mu_{t}= \begin{cases}\mu_{0} e^{(\rho+\alpha) t} & , \quad t \in[0, \hat{\tau}) \\ -\zeta^{-1}\left[\bar{p}_{e}-\tilde{p}_{t}\right] & , \quad t \in[\hat{\tau}, \tilde{\tau}) \\ 0 & , \quad t \in[\tilde{\tau}, \infty)\end{cases}$

$v_{t}= \begin{cases}0 & , \quad t \notin[\hat{\tau}, \tilde{\tau}) \\ \zeta^{-1}\left[(\rho+\alpha)\left(\bar{p}_{e}-\tilde{p}_{t}\right)+\dot{\tilde{p}}_{t}\right], & t \in[\hat{\tau}, \tilde{\tau})\end{cases}$ 
B.2 Let there exist $\lambda_{0}, \mu_{0}, \delta, \widetilde{\tau}, \tilde{\tau}$ and $\tilde{\theta}$ such that (A1)-(A3) are satisfied and the solution is given by (A8). The same remarks as in B.1 suggest that the transversality conditions are satisfied. The values of the dual variables $\gamma_{a t}$, $\gamma_{e t}, \gamma_{r t}, \mu_{t}$ and $v_{t}$ for which all the first order conditions (2)-(12) hold are now given by:

$\gamma_{a t}=\left\{\begin{array}{lll}c_{a}+\mu_{0} e^{(\rho+\alpha) t} & , & t \in[0, \delta) \\ 0 & , \quad t \in[\delta, \widetilde{\tau}) \\ c_{a}-\zeta^{-1}\left[\bar{p}_{e}-\tilde{p}_{t}\right] & , \quad t \in[\widetilde{\tau}, \tilde{\tau}) \\ c_{a} & , \quad t \in[\widetilde{\tau}, \infty)\end{array}\right.$

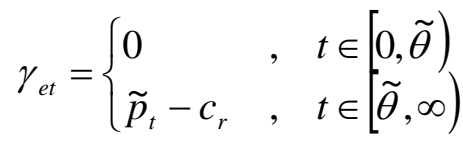

$\gamma_{r t}= \begin{cases}c_{r}-\hat{p}_{t} & , \quad t \in[0, \delta) \\ c_{r}-\breve{p}_{t} & , \quad t \in[\delta, \widetilde{\tau}) \\ c_{r}-\bar{p}_{e} & , \quad t \in[\widetilde{\tau}, \tilde{\tau}) \\ c_{r}-\tilde{p}_{t} & , \quad t \in[\tilde{\tau}, \tilde{\theta}) \\ 0 & , \quad t \in[\tilde{\theta}, \infty)\end{cases}$

$\mu_{t}= \begin{cases}\mu_{0} e^{(\rho+\alpha) t} & , \quad t \in[0, \delta) \\ -C_{a} & , \quad t \in[\delta, \widetilde{\tau}) \\ -\zeta^{-1}\left[\bar{p}_{e}-\tilde{p}_{t}\right] & , \quad t \in[\tilde{\tau}, \tilde{\tau}) \\ 0 & , \quad t \in[\tilde{\tau}, \infty)\end{cases}$

$v_{t}= \begin{cases}0 & , \quad t \notin[\delta, \tilde{\tau}) \\ (\rho+\alpha) c_{a} & , \quad t \in[\delta, \tilde{\tau}) \\ \zeta^{-1}\left[(\rho+\alpha)\left(\bar{p}_{e}-\tilde{p}_{t}\right)+\dot{\tilde{p}}_{t}\right] & , \quad t \in[\tilde{\tau}, \tilde{\tau})\end{cases}$ 


\section{References}

Amigues, J.P., Favard P., Gaudet G., Moreaux M., 1998. On the optimal order of natural resource use when the capacity of the inexhaustible substitute is constrained, Journal of Economic Theory, 80, 153-170.

Chakravorty, U., Roumasset J., Tse K., 1997. Endogenous substitution among energy resources and global warming, Journal of Political Economy, 106, 1201-1234.

Chakravorty, U., Magné B., Moreaux M., 2003. Plafond de concentration atmosphérique en carbone et substitutions entre ressources énergétiques, forthcoming in Annales d'Economie et de Statistique.

Chakravorty, U., Magné B., Moreaux M., 2005. Could nuclear power solve the global warming problem? Unpublished Manuscript, University of Toulouse.

Farzin, Y.H., 1996. Optimal pricing of environmental and natural resource use with stock externalities, Journal of Public Economics, 62, 31-57.

Fisher, C., Toman M., Withagen C., 2004. Optimal investment in clean production capacity, Environmental and Resource Economics 28(3), 325-345.

Forster, B.A., 1980. Optimal energy use in a polluted environment, Journal of Environmental Economics and Management, 7, 321-333.

Gjerde, J. Grepperud, S., Kverndokk, S., 1999. Optimal climate policy under the possibility of a catastrophe, Resource and Energy Economics, 21(3-4), 289-317

Herfindahl, O. L., 1967. Depletion and economic theory, in M. Gaffney, ed., Extractive Resources and Taxation, Madison : University of Wisconsin Press, 63-90.

Hoel, M., Kverndokk S., 1996. Depletion of Fossil Fuels and the Impacts of Global Warming, Resource and Energy Economics, 18, 115-136.

Hotelling, H., 1931. The economics of exhaustible resources, Journal of Political Economy, 39, 137-175.

Kolstad, C.D., Kraukraemer J.A., 1993. Natural resource use and the environment in Handbook of Natural Resource and Energy Economics, Vol. III, Chap. 26, edited by A.V. Kneese and J.L. Sweeney, Elsevier Science Publishers.

Lutz, W., Sanderson, W., Scherbov, S., 2001. The end of world population growth, Nature, 412, $543-546$.

Manne, A.S., Richels, R.G., 1991. Global CO2 emission reductions - the impact of rising energy costs, Energy Journal 12(1), 87-107. 
McKibbin, W.J., Wilcoxen, P.J., 2002. The role of economics in climate change policy, Journal of Economic Perspectives 16(2), 107-129.

Nordhaus, W.D., Yang, Z.,1996. A Regional Dynamic General Equilibrium Model of Alternative Climate Change Strategies, American Economic Review, 86(4), 741-765.

Sinclair, P., 1994. On the optimal trend of fossil-fuel taxation, Oxford Economic Papers, 46, 857868.

Tahvonen, O., 1997. Fossil fuels, stock externalities, and backstop technology, Canadian Journal of Economics, 30, 855-874.

Toman, M., Withagen, C., 2000. Accumulative pollution, 'clean technology', and policy design, Resource and Energy Economics, 22, 367-384.

Ulph, A., Ulph, D, 1994. The optimal time-path of a carbon tax. Oxford Economic Papers, 46, 857-868.

United Nations, 2002. World Population Prospects: The 2002 Revision, Highlights. United Nations publications.

Zhang, Z.X., Folmer, H., 1998. Economic modelling approaches to cost estimates for the control of carbon dioxide emissions, Energy Economics 20(1), 101-120. 


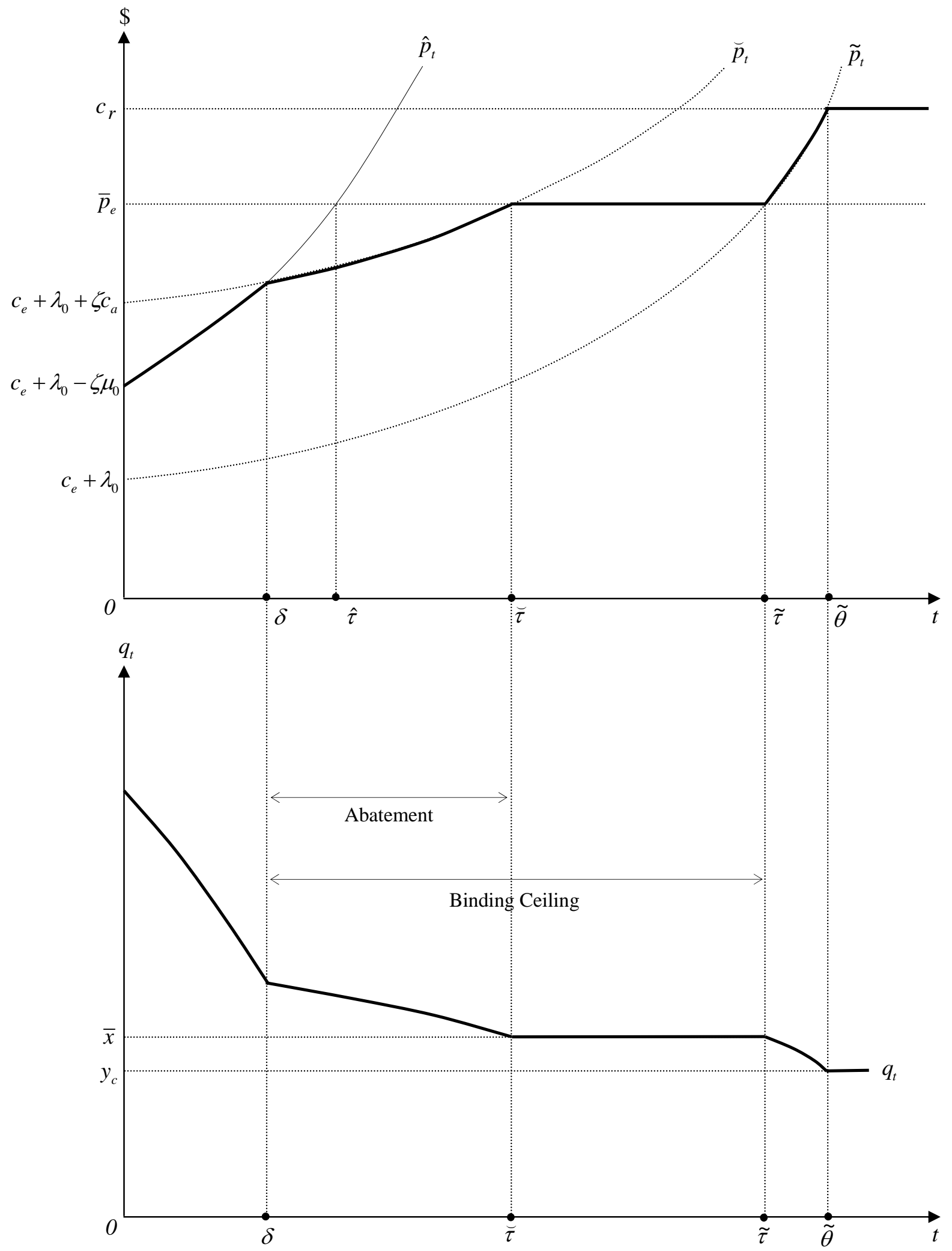

Fig. 1. Stationary Demand with $\bar{p}_{e}<C_{r}$ : Only Coal is used at the Ceiling 

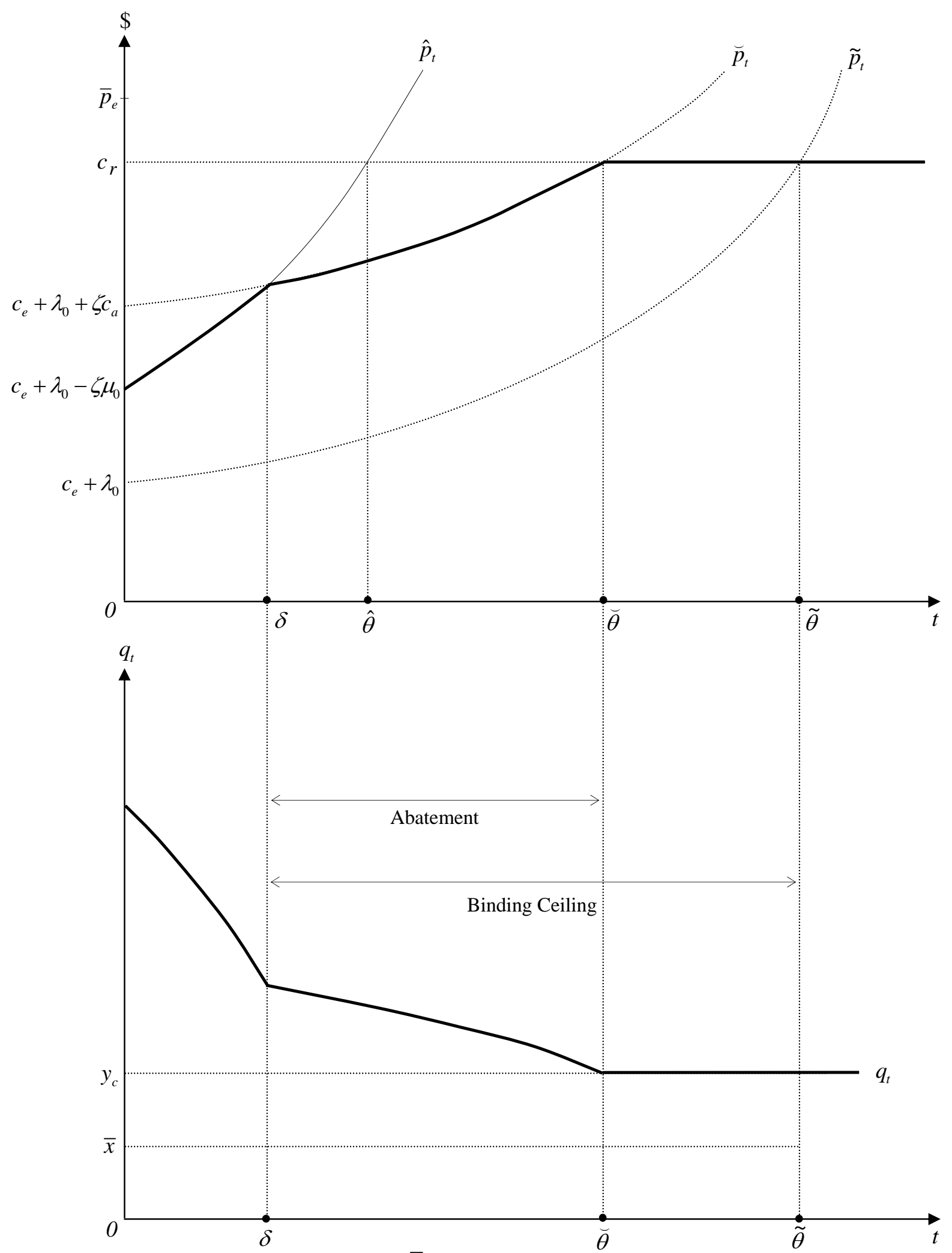

Fig. 2. Stationary Demand with $\bar{p}_{e}>c_{r}$ : Both Resources are used at the Ceiling 


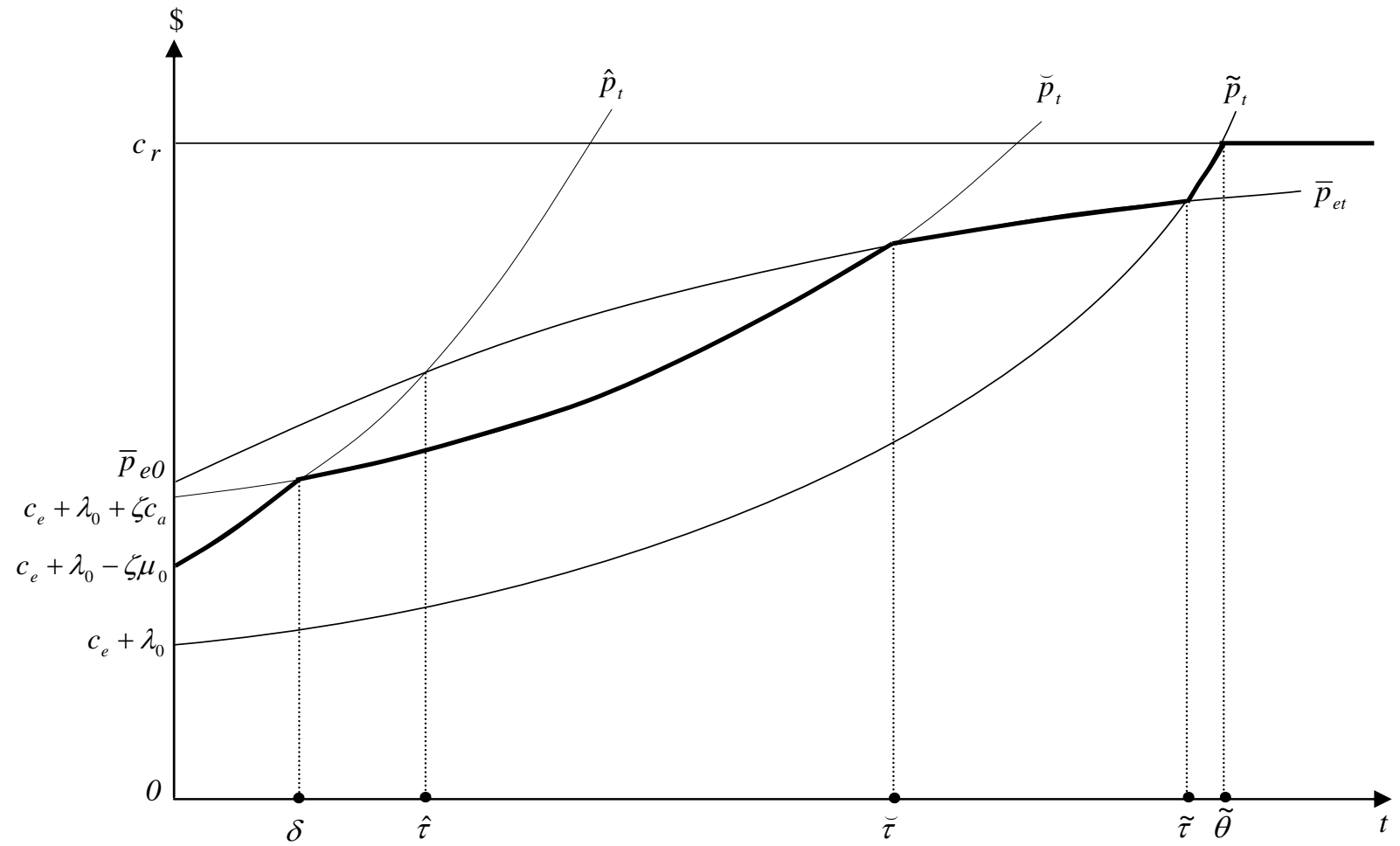

Fig. 3. Increasing Demand with $\bar{p}_{e \infty}<c_{r}$ : Abatement Period at the Beginning of the Ceiling

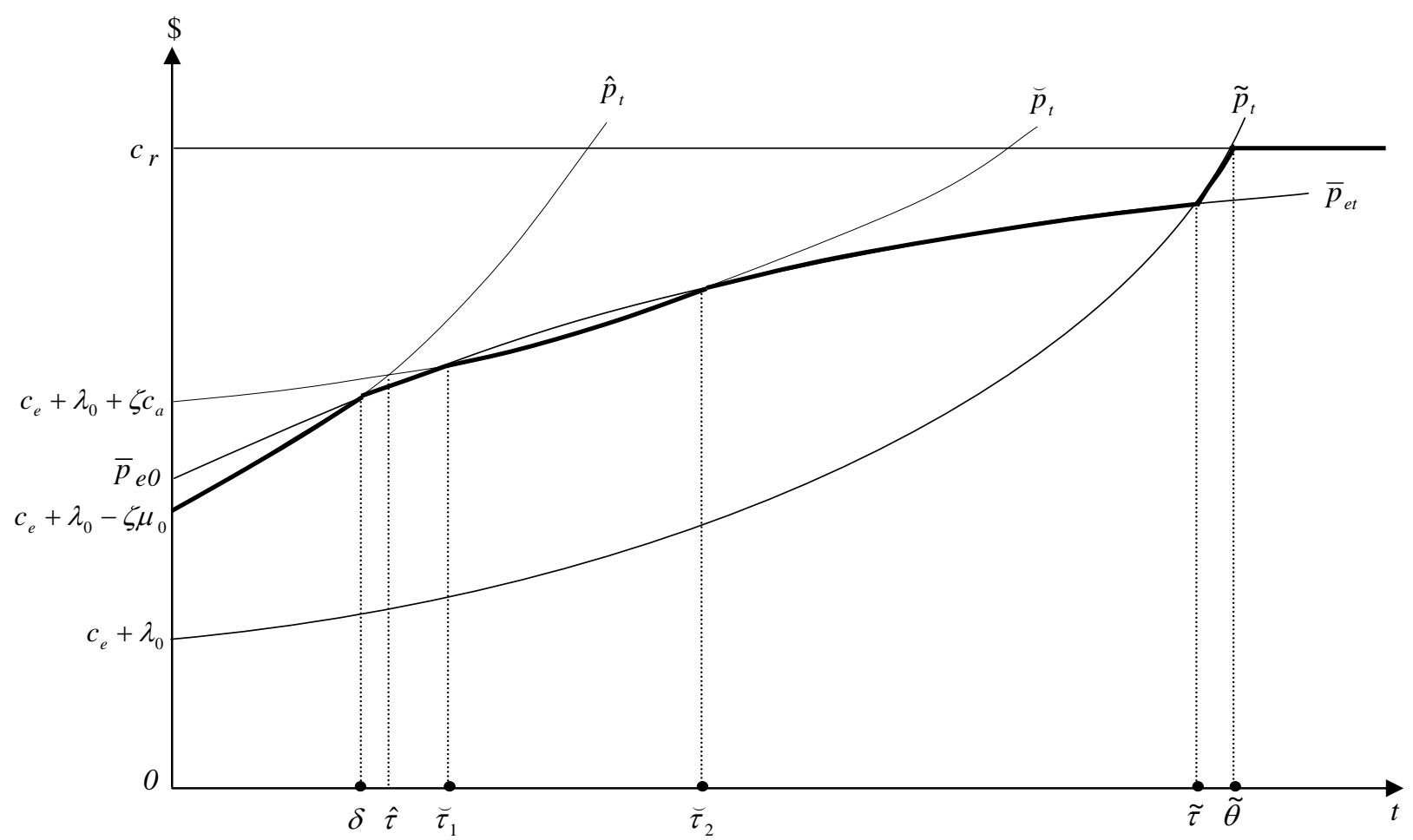

Fig. 4. Increasing Demand with $\bar{p}_{e \infty}<c_{r}$ : Abatement Strictly in the Ceiling Interior 


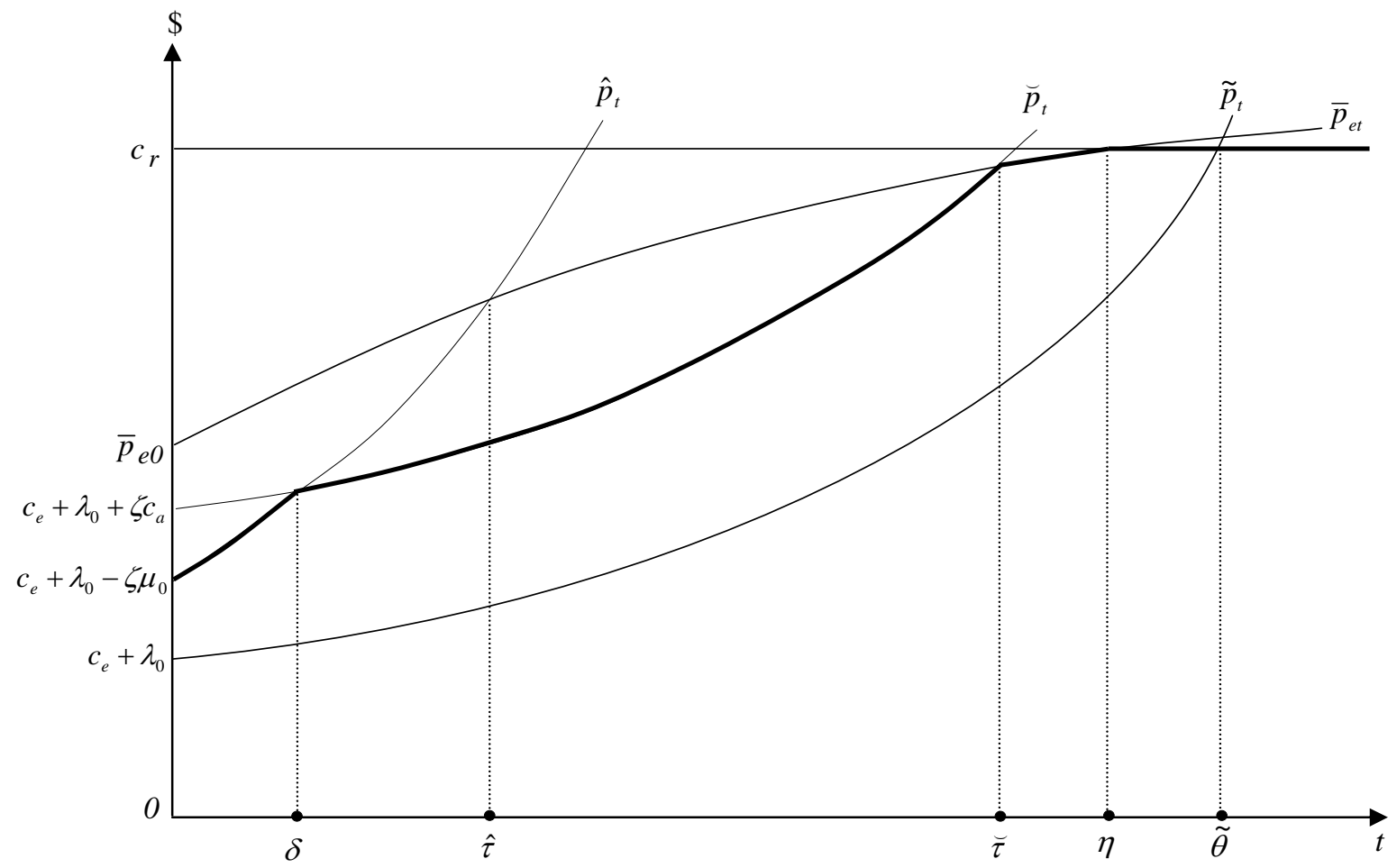

Fig. 5. Increasing Demand with $\bar{p}_{e 0}<c_{r}<\bar{p}_{e \infty}$ : Abatement at the Beginning of the Ceiling

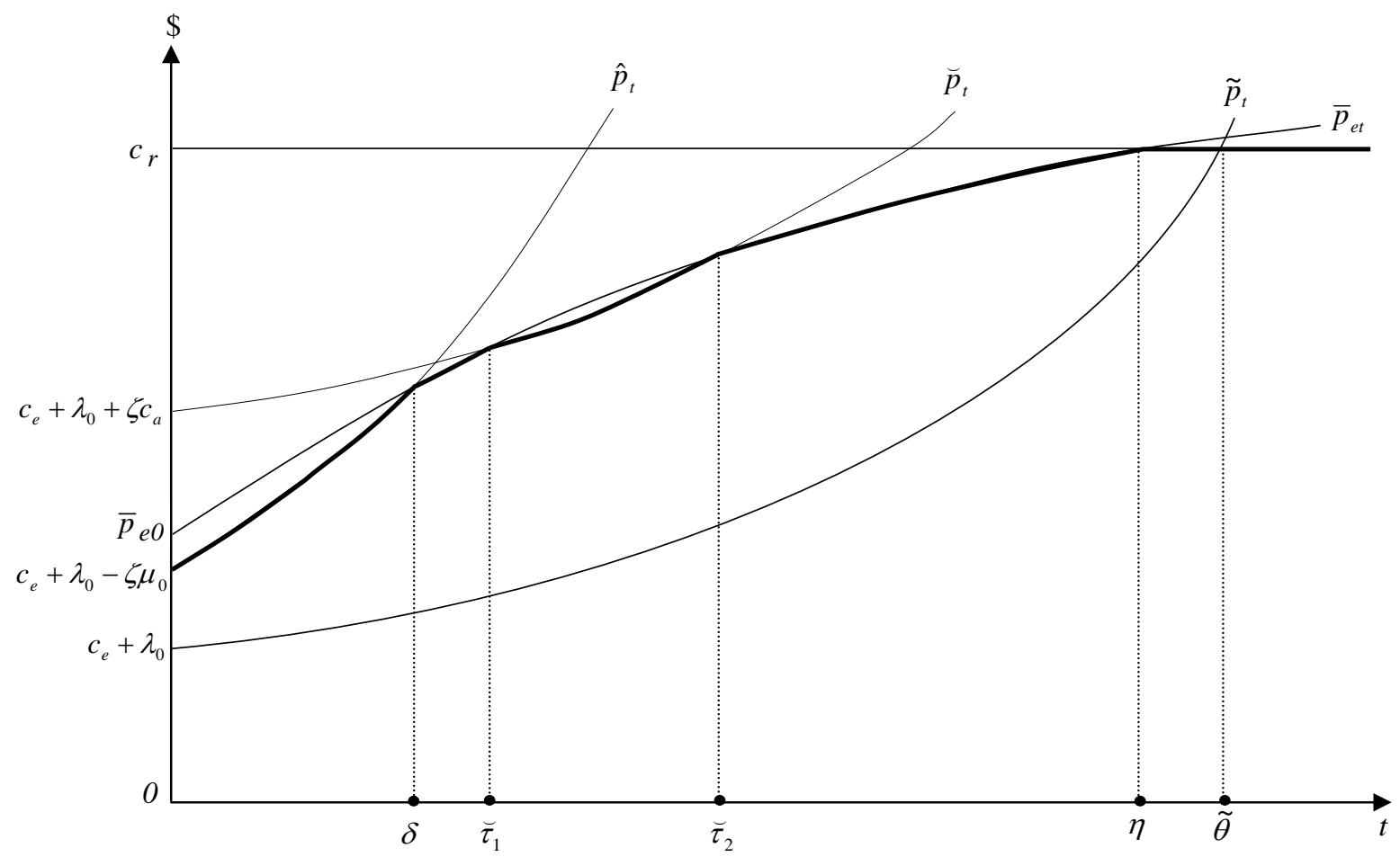

Fig. 6. Increasing Demand with $\bar{p}_{e 0}<c_{r}<\bar{p}_{e \infty}$ : Abatement Strictly in the Ceiling Interior 


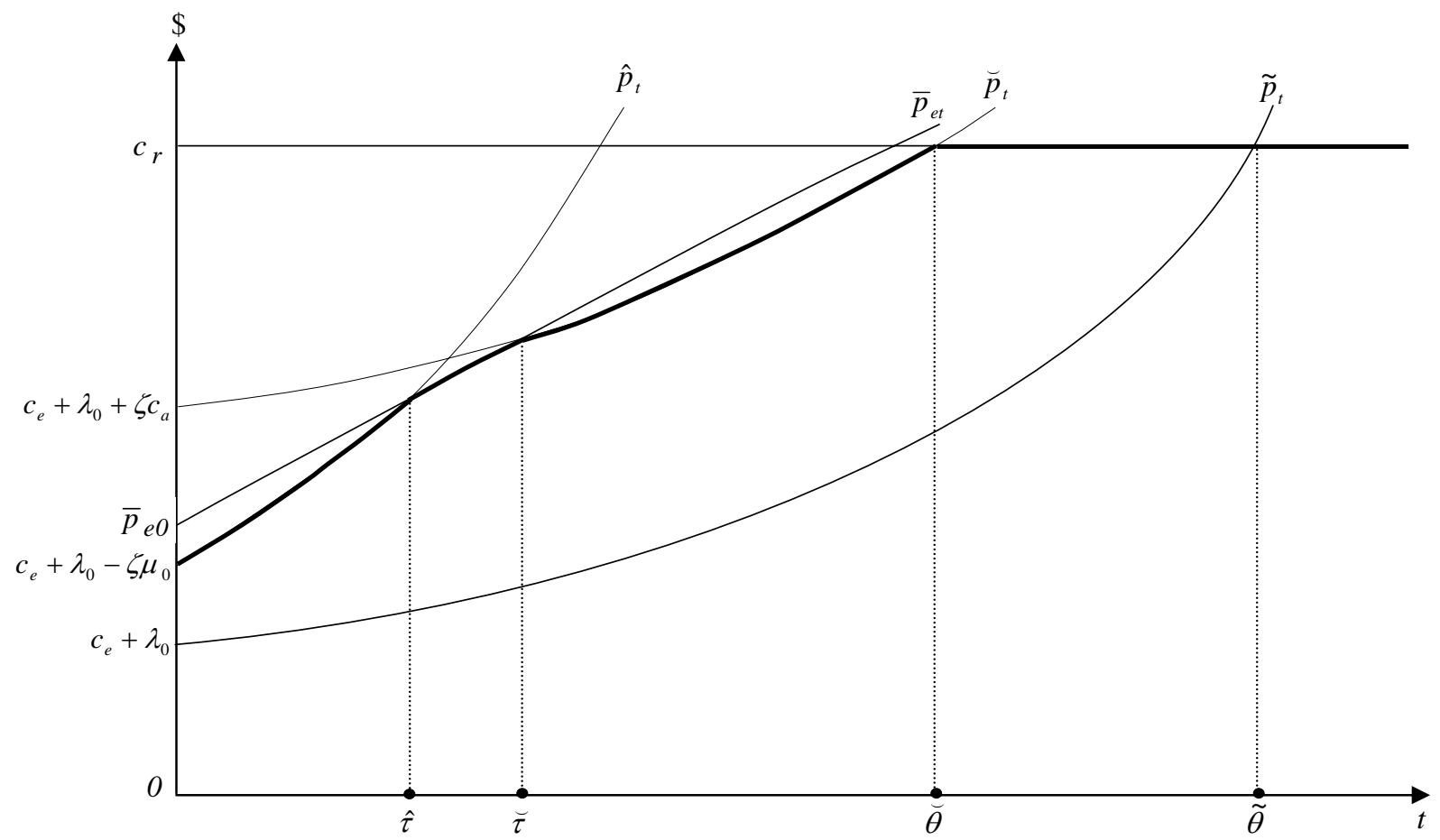

Fig. 7. Increasing Demand with $\bar{p}_{e 0}<C_{r}<\bar{p}_{e \infty}$ : Abatement at the End of the Ceiling

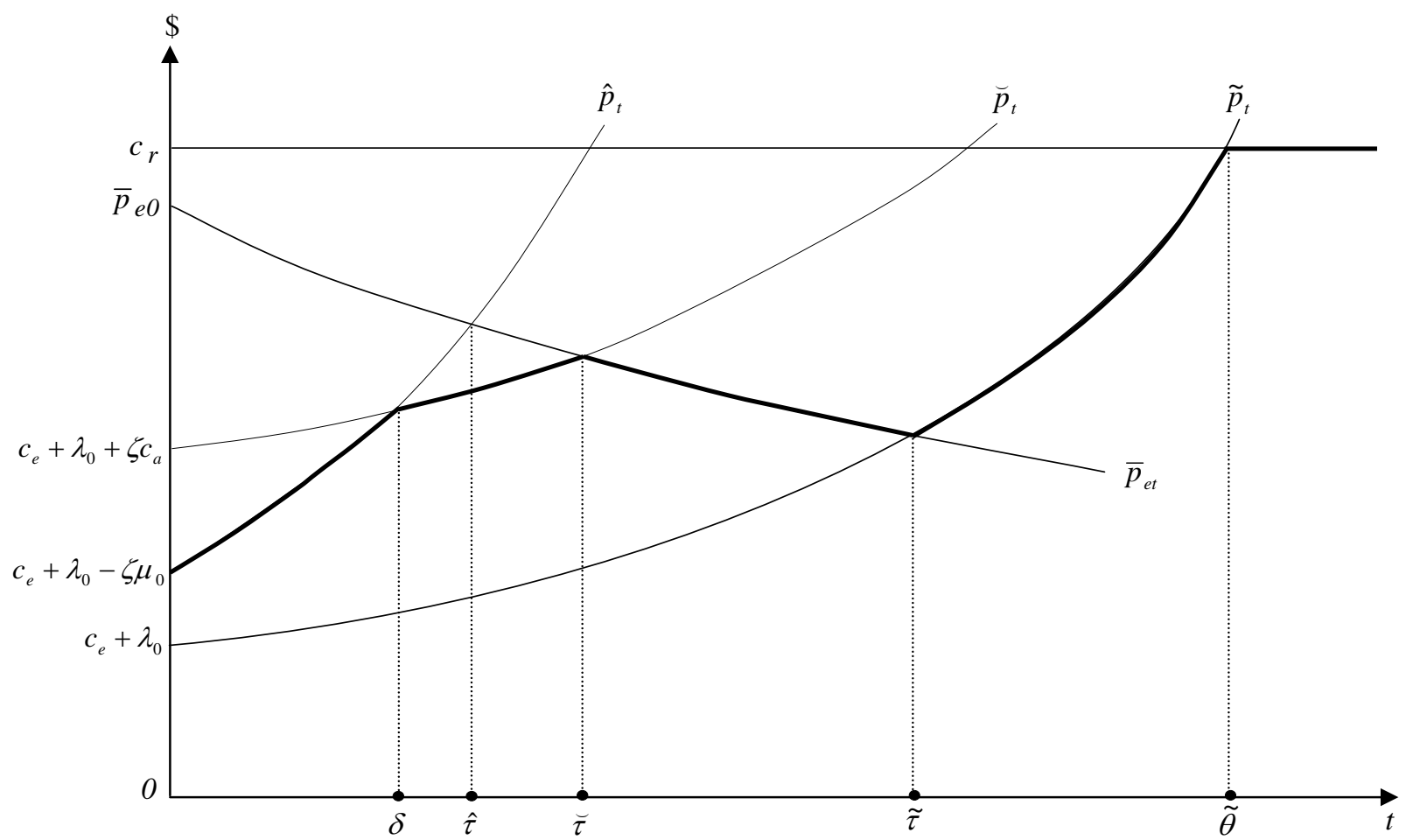

Fig. 8. Decreasing Demand with $\bar{p}_{e 0}<c_{r}$ : Only Coal is used at the Ceiling 


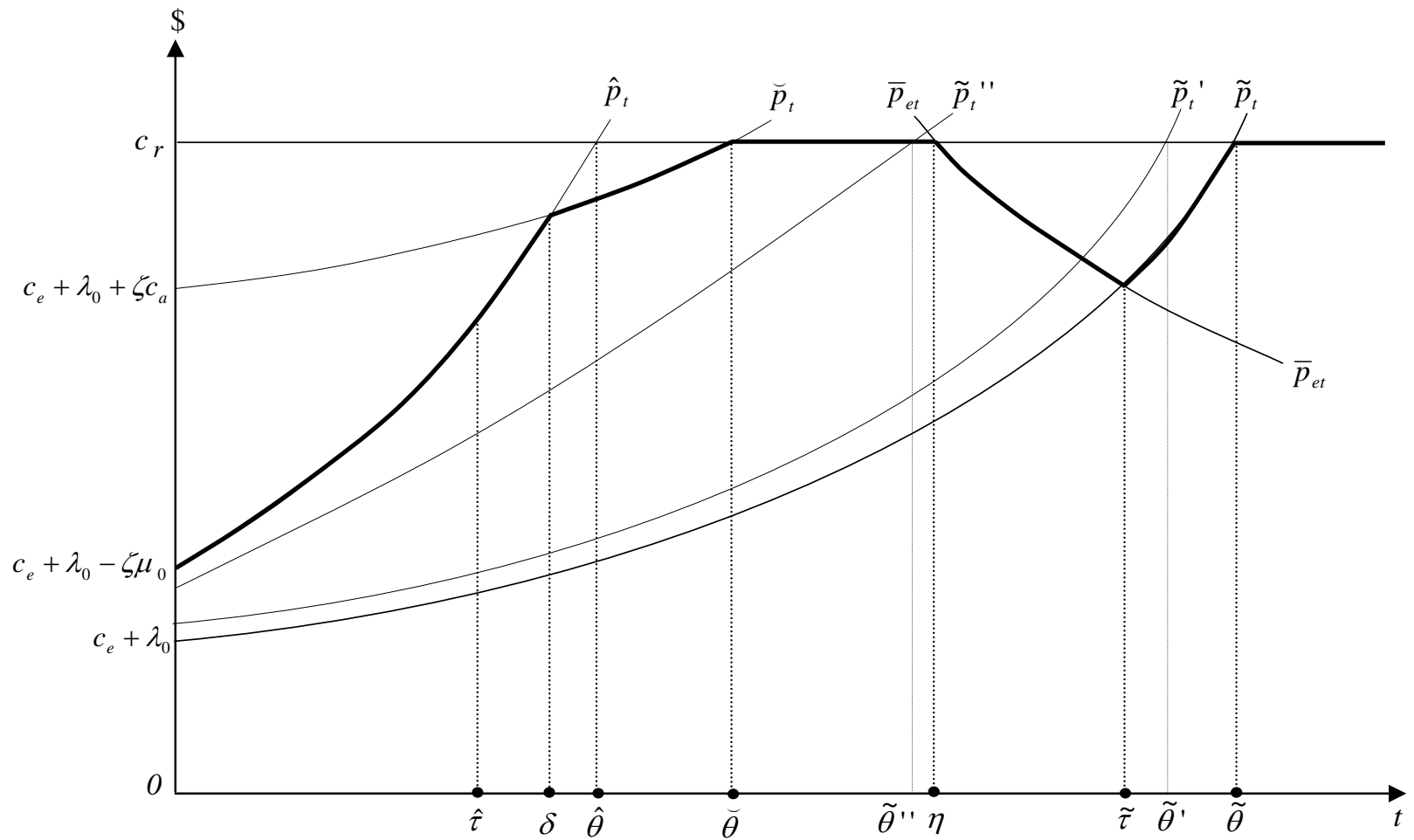

Fig. 9. Decreasing Demand with $\bar{p}_{e 0}>c_{r}>\bar{p}_{e \infty}$ : Solar Energy is used during two Disjoint Time Periods 